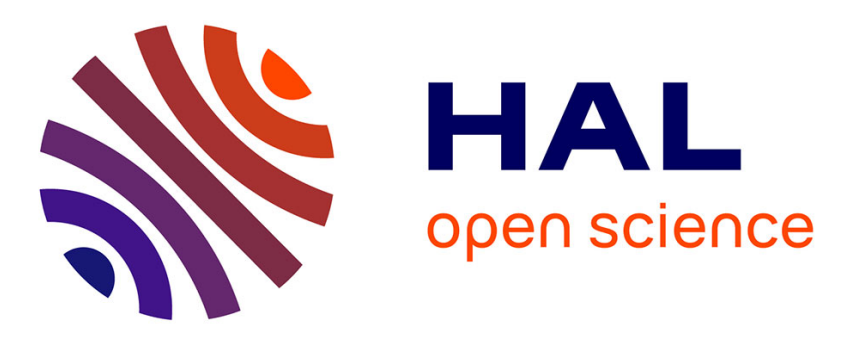

\title{
Actuator line method applied to grid turbulence generation for large-Eddy simulations
}

Félix Houtin-Mongrolle, L. Bricteux, Pierre Benard, G. Lartigue, Vincent Moureau, J. Reveillon

\section{- To cite this version:}

Félix Houtin-Mongrolle, L. Bricteux, Pierre Benard, G. Lartigue, Vincent Moureau, et al.. Actuator line method applied to grid turbulence generation for large-Eddy simulations. Journal of Turbulence, 2020, 10.1080/14685248.2020.1803495 . hal-02915062

\section{HAL Id: hal-02915062 \\ https://hal.science/hal-02915062}

Submitted on 26 Nov 2020

HAL is a multi-disciplinary open access archive for the deposit and dissemination of scientific research documents, whether they are published or not. The documents may come from teaching and research institutions in France or abroad, or from public or private research centers.
L'archive ouverte pluridisciplinaire HAL, est destinée au dépôt et à la diffusion de documents scientifiques de niveau recherche, publiés ou non, émanant des établissements d'enseignement et de recherche français ou étrangers, des laboratoires publics ou privés. 


\title{
Actuator line method applied to grid turbulence generation for Large-Eddy Simulations
}

\author{
F. Houtin-Mongrolle ${ }^{1, a}$, L. Bricteux ${ }^{2}$, P. Benard $^{1}$, \\ G. Lartigue ${ }^{1}$, V. Moureau ${ }^{1}$, J. Reveillon $^{1}$ \\ ${ }^{1}$ Normandie Univ, INSA Rouen, UNIROUEN, CNRS, CORIA, Rouen 76000, France \\ ${ }^{2}$ University of Mons (UMONS), Polytechnic Faculty, Belgium \\ ${ }^{a}$ Corresponding author: felix.houtin-mongrolle@coria.fr \\ Preprint version*
}

\begin{abstract}
The constant growth of computational resources allows performing Large-Eddy Simulation (LES) on realistic flow configurations. In this context, it is important to properly model boundary conditions and particularly inflow turbulence. This study addresses wind tunnel applications where the object under investigation is downstream of a turbulence grid. This grid aims at generating a highly turbulent sheared flow for further use in wind turbines applications. In this work, an original strategy based on the actuator line method (ALM) is proposed to emulate the grid and generate realistic flow structures downstream at a moderate computational cost. As a first validation test of the proposed method, the LES of a single square rod is performed and the results are compared to a scale resolving simulation. In a second phase, the method is applied to a passive grid with non-homogeneous spacing, which generates fully-developed turbulence. Results are compared to experimental data and other state-of-the-art turbulence injection strategies such as homogeneous isotropic turbulence injection and also scale resolving simulations.
\end{abstract}

Keywords: LES ; Actuator Line Method ; Grid Turbulence

\section{Introduction}

\subsection{Context}

This paper deals with Large-Eddy Simulation (LES) of turbulence generation in wind tunnels. Numerical simulation of turbulence is widely used in the fields of wind energy and aeronautics for various applications such as turbine blade design, turbofans rotors study, wind farm wake interference, wind turbine siting, etc. For all these applications, predictive numerical methods that reproduce accurately the flow physics are required.

Verification and validation of numerical codes efforts have become more and more important in CFD [1]. Wind tunnels experiments are widely used to validate numerical methods and assess

*Accepted for publication in Journal of Turbulence (21 Jul 2020). 
the quality of simulation results. However, these measurements are significantly impacted by the turbulence generated upstream of the region of interest. It is thus important to reproduce with high fidelity the turbulent flow generated at the test section entry of a wind tunnel. The numerical inflow turbulence must indeed feature the same velocity profiles, turbulence intensity and spectral content as the one produced in the wind tunnel.

There are many ways to generate turbulence in a wind tunnel. A recent and synthetic review of these techniques can be found in the paper of Vita et al. [2]. According to literature [2 4], turbulence grid generation is the most effective and reliable technique to produce turbulence in a wind tunnel. These grids can be classified in three categories: passive grids, active grids and fractal grids. Active grid generators aim at controlling the injected turbulence using moving elements, fans or jets. These methods require a careful tuning to obtain the desired effect on the flow and can require expensive hardware. Passive grids are far less expensive and are most commonly used. The present paper focuses only on this kind of turbulence generation. Passive grids [5] are mainly constituted of arrays of round or square rods, wires, airfoils, etc. Recent works [6.7] also demonstrate the interest of passive fractal grids that yield higher turbulence levels, shorter development lengths and also allow to finely tune turbulence intensity and pressure drop across the grid. Numerical simulation of fractal grid generated turbulence has been investigated [8] by performing direct numerical simulation (DNS) using a high-order code.

\subsection{Passive grid turbulence numerical generation methods}

Turbulence injection in unsteady simulations is a field of interest since decades. Many methods have already been developed and extensive reviews of those methods can be found in the literature [9 11]. Turbulent inflow generation methods can be categorized into synthetic and precursor turbulence methods [10].

\subsubsection{Synthetic turbulence}

The simplest method to generate turbulence at the domain inlet is to superpose a random noise to the inlet boundary condition. However, a random noise does not feature the characteristics of real turbulence. Even if the energy spectrum of the random noise matches that of developed turbulence, it cannot reproduce the other features such as Reynolds stress equilibrium or Kolmogorov energy cascade. As a consequence, this type of turbulence is rapidly damped by the Navier-Stokes solver. In order to reproduce some of the features of developed turbulence, one can generate an isotropic synthetic velocity field using random Fourier modes such that the velocity fluctuations at position $\mathbf{x}$ in the inlet plane reads

$$
\mathbf{u}^{\prime}(\mathbf{x})=\sum_{n=1}^{N} 2 \hat{u}_{n} \cos \left(\kappa_{n} \cdot \mathbf{x}+\psi_{n}\right) \sigma_{n},
$$

where $\hat{u}_{n}, \psi_{n}$ and $\sigma_{n}$ are the amplitude, phase and direction of the mode $n$, respectively. The wave number vector for each mode $n$ is $\kappa_{n}$. This method requires to prescribe the energy spectrum and the turbulent length scale. To enforce time coherence from independent realizations of $\mathbf{u}^{\prime}$, one uses a causal time filter [12,13]. The anisotropy of the synthesized turbulence is introduced [14] using a prescribed Reynolds stress tensor $R_{i j}$ to produce anisotropic turbulence. This technique is easy to implement and is computationally cheap. Yet, Fourier techniques only generate one random phase angle for each wavenumber magnitude $\kappa_{n}$, the Fourier plane is thus discretized with sparse points located on circles of radius $\kappa_{n}$ (one point per circle), instead of being uniformly sampled on a grid. As a consequence, the generated structures have an unrealistic spectral content. This can be cured using precursor methods as described hereafter. The digital filter method constitutes an improvement to this method as it allows generating 
filtered random structures that features realistic spatial and temporal correlations. The generated data can also be further transformed with a prescribed Reynolds stress tensor in order to obtain anisotropic flows.

\subsubsection{Precursor methods}

Precursor methods require to compute a separate turbulence database that is further introduced at the inlet of the computational domain. At each time step, a plane including the velocity field is extracted from the turbulence database and is mapped onto the inflow plane. The inflow conditions can be issued from another LES or DNS simulation (e.g. homogeneous isotropic turbulence, channel flow or turbulent boundary layer) or from a particular algorithm that generates realistic velocity fields (e.g. Mann algorithm [15]). The purpose of the Mann algorithm is to generate realistic atmospheric turbulent inflows. It provides a model of the spectral tensor of neutral atmospheric surface layer turbulence. This model uses rapid distortion theory and eddy-lifetime assumption to produce anisotropic turbulence. However, it requires the generation of large pre-computed turbulence databases that are periodically inserted in the computational domain and requires large memory space when the simulations span long time periods. The periodic character of this method leads to artificial periodicity effects on the flow. This further motivates an approach avoiding these periodicity effects. In both cases, precursor methods feature in general a higher quality compared to synthetic inflows: better spectral behavior, realistic turbulence length, correct turbulence intensity... A particular care is also required when the wind direction changes [16].

Recycling method [14,17] can be used as well. Indeed recycling method intent to generate fully turbulent flow by developing near wall boundary layers. The boundary layers are developed through re-injection of the flow fluctuations inside the domain to the domain inlet [18,19]. The drawbacks of such method is the requirement to have a larger computational domain and it is only applicable to boundary layer flows.

\subsubsection{Resolved grid flow}

A realistic strategy to simulate grid turbulence consists in explicitly discretizing the bodies which generate the turbulence. The main drawback of this method is the computational cost as wall turbulence has to be fairly resolved, whatever the LES approach (wall resolved or wall modeled). Moreover, some sharp devices may lead to meshing difficulties, low-quality cells and the necessity to use unstructured meshes. Considering the high Reynolds number of wind tunnel flows, this method is of little applicability. However, it will be used in this paper to validate the proposed approach.

An immersed boundary method [20 22] constitutes an interesting alternative that avoids the discretization of the geometry. This method is mainly used on structured meshes to take into account complex geometry and on unstructured or structured meshes to emulate the flow around a moving object. Yet particular care has to be taken to resolve the boundary layers.

\subsection{A novel approach to synthesize grid turbulence in a computa- tional domain}

It is proposed here to demonstrate that an original method based on the Actuator Line Method [23] is suitable to emulate the effect of a turbulence grid in a wind tunnel. It is shown here that the generated turbulence features very similar physical properties (Strouhal number, turbulence intensity, spectral behavior) compared to the approach consisting in discretizing the rods of the grid. The proposed method results in a much lower computational cost as it doesn't require to explicitly discretize the turbulence generating rods. The paper 
is structured as follows. Section 2 describes the governing flow equation, the standard actuator line method (ALM) and the numerical method. Section 3 presents and evaluates the new ALM-based turbulence generation approach, named dynamic actuator line method (DALM), on a rod wake case. Section 4 presents the turbulence generated by a grid in a wind tunnel with several numerical approaches (the DALM, a resolved flow with body-fitted grid and a precursor method based on homogeneous isotropic turbulence (HIT) injection) and compares them to experimental results. The last section 5 summarizes all theses results and proposes further perspectives.

\section{Governing equations and numerical method}

\subsection{Governing equations}

The governing equations are the Navier-Stokes equations for incompressible flow, supplemented by a sub-grid scale (SGS) model. The truncation operator, which consists in projecting a field onto the LES grid, is written as $\tilde{\bullet}$. Using this notation, the evolution equations for the LES velocity field are formally written as:

$$
\begin{aligned}
& \nabla \cdot \widetilde{\mathbf{u}}=0 \\
& \frac{\partial \widetilde{\mathbf{u}}}{\partial t}+(\widetilde{\mathbf{u}} \cdot \nabla) \widetilde{\mathbf{u}}=-\nabla \widetilde{P}+v \nabla^{2} \widetilde{\mathbf{u}}+\nabla \cdot \widetilde{\tau}^{M}+\mathbf{f},
\end{aligned}
$$

where $\widetilde{P}=\frac{1}{\rho} \tilde{p}$ is the reduced pressure field, $\nu$ the kinematic viscosity, and $\widetilde{\tau}^{M}$ the modeled SGS stress tensor. The body force $\mathbf{f}$ is introduced here to model the effects of the rod array on the flow in the ALM approach and will be detailed in the following subsections.

In this study, the sub-grid Reynolds stress tensor is modeled using the $\sigma$-model [24]. This model ensures the proper near-wall behavior in turbulent boundary layers as well as a low SGS dissipation level in vortical regions of the flow.

\subsection{Actuator Line Method}

The method developed in this paper is based on the standard ALM [23], initially designed for horizontal-axis wind turbine wakes modeling. In such model, the unsteady force distribution of a blade on the flow is prescribed instead of being resolved by the flow solver. This body force $\mathbf{f}$ added to the momentum balance equation is distributed along lines representing the aerodynamic loads of each rotating blades of the rotor [23,25]. Thus, this technique provides an interesting trade-off between computational cost and precision for flows at high Reynolds number.

Following the formulation of Peet et al. [25], the blades are divided into discrete elements. The local lift $L$ and drag $D$ forces acting on each element are computed using the blade element theory of Glauert [26]:

$$
\begin{aligned}
L & =\frac{1}{2} \rho u_{r e l}^{2} l w C_{L}(\alpha), \\
D & =\frac{1}{2} \rho u_{r e l}^{2} l w C_{D}(\alpha),
\end{aligned}
$$

where $C_{L}$ and $C_{D}$ are the lift and drag coefficients of the rod, $\alpha$ is the local angle of attack, $u_{r e l}$ is the local velocity relative to the geometry, $l$ is a local length, i.e. the chord of the emulated profile, and $w$ is the actuator element width, i.e. the distance between two actuator elements. For a wind tunnel static grid, each actuator element use the unperturbed incoming velocity 

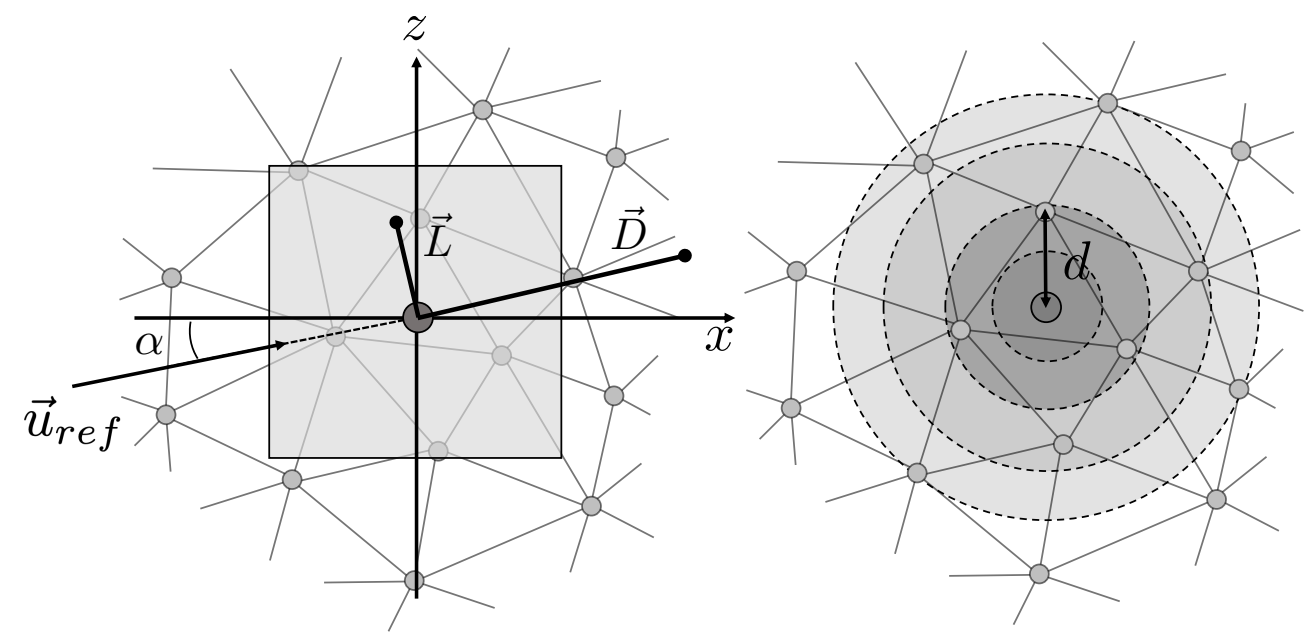

Figure 1: Description of the forces computations and mollification process on one rod element (rod is aligned along y direction).

and its related angle of attack. The body force is projected onto the Eulerian grid using a mollification function [25]27] $\eta_{\epsilon}$, defined as:

$$
\eta_{\epsilon}(d)=\frac{1}{\epsilon^{3} \pi^{3 / 2}} \exp \left[-\left(\frac{d}{\epsilon}\right)^{2}\right]
$$

$d$ being the distance between a grid node and the element position and $\epsilon$ the mollifier width parameter. To ensure a conservative treatment of the forces on a numerical grid with a given cell size $h, \epsilon / h$ has to be set such that:

$$
\int_{R^{3}} \eta_{\epsilon}(\mathbf{x}) \mathrm{d} \mathbf{x}=1
$$

A lower bound limit has been identified in previous studies [23|28] as $\epsilon / h \geq 2$. In this work, the mollifier width is set to the optimal value determined by Martinez-Tossas et al. [29] $\epsilon=0.2 l$, $l$ being the body characteristic size, obtained on a static airfoil configuration. Therefore the minimum resolution near the DALM should be $l / h=10$, i.e. ten cells per geometry section length. This technique provides a smooth force $\mathbf{f}$ distributed on the grid obtained from the forces concentrated on a given element depicted in Fig. 1. Finally, the body force source term $\mathbf{f}$ at a position $\mathbf{x}$ in the momentum balance equation reads:

$$
\mathbf{f}(\mathbf{x})=-\sum_{k=1}^{N}\left(L_{k} \mathbf{e}_{L}+D_{k} \mathbf{e}_{D}\right) \eta_{\epsilon}\left(\left\|\mathbf{x}-\mathbf{x}_{k}\right\|\right),
$$

where $N$ is the number of actuator points along the span of the geometry in order to respect the mollifier width, $\mathbf{e}_{L}$ and $\mathbf{e}_{D}$ are the unit lift and drag vectors.

\subsection{YALES2 flow solver}

The YALES2 [30] flow solver is used here. It is a massively-parallel finite-volume solver, which is specifically tailored for Large-Eddy Simulation, and relies on a central 4th-order numerical scheme for spatial discretization, and a 4th-order Runge-Kutta method [31] for the time integration. The discretization is formally fourth-order on Cartesian grids. On regular tetrahedral grids, i.e. with a homogeneous control volume size, the spatial errors are also fourth-order but this order of convergence can decrease on low quality grids as classical finite volumes methods 
do. The fairly high order achieved here features low numerical dispersion and dissipation errors [27]. Hence, it ensures the proper transport of fine vortical structures on a grid. This code solves the incompressible and low-Mach number Navier-Stokes equations for turbulent flows on structured or unstructured meshes using a projection method for pressure-velocity coupling [32]. The Poisson equation in the projection method is solved with a high-performance deflated preconditioned conjugate gradient solver [33. Combined with an in-house hierarchical grid partitioning with up to three-level in hybrid MPI/OpenMP mode, the code can handle grids with several billions of elements [34]. The ALM framework has already been developed and validated into the YALES2 solver [27].

\section{A new actuator line method-based turbulence gener- ation approach}

The full resolution of the geometrical details of the wind tunnel turbulence grid constitutes the highest level of fidelity to reproduce the same turbulent inflow as that produced in a wind tunnel. Yet, such simulation leads to prohibitive computational costs due to the high resolution needed to resolve or model the boundary layers and the wakes released by the turbulence generators. To alleviate this cost, an original method called dynamic actuator line method (DALM), derived from the standard ALM, is presented in this section. This method and the standard actuator line method will be compared in a test case consisting of the turbulent flow around a square rod similar to those found in wind tunnel applications.

\subsection{Reference case results}

The square rod flow test case considered here to validate the approach is well documented in the literature. Indeed, aerodynamic experimental [35 37] data for the flow over a square rod at a fairly high Reynolds number $\left(R e=\mathcal{O}\left(10^{4}\right)\right)$ have been gathered and are summarized in Tab. 1, which provides time-averaged lift and drag coefficients $\left\langle C_{L}\right\rangle,\left\langle C_{D}\right\rangle$ as well as their RMS values $\left\langle C_{L}^{\prime 2}\right\rangle^{1 / 2},\left\langle C_{D}^{\prime}{ }^{2}\right\rangle^{1 / 2}$. The Strouhal number $S t$ is defined as $S t=f l / u_{\text {ref }}$, where $f$ is the frequency of the vortex shedding in the wake, $u_{r e f}$ is the reference velocity and $l$ a reference length scale, chosen here as the side length of the rod. For the LES considered here, the flow regime is such that $R e=\frac{u_{r e f} l}{\nu}=3.0 \times 10^{4}$ where the reference velocity $u_{r e f}$ is the inlet velocity. The computational domain and the rod geometry are displayed in Fig. 2 . According to the work of Fukomoto et al. [38] the choice of $3 l$ in the spanwise direction is sufficient to estimate properly the aerodynamic forces fluctuations. A periodicity along the rod span axis is set on the side boundaries. Slip wall conditions are used for the upper and lower boundaries, and they are far enough to have a negligible effect on the flow. A wall model [39] is applied on the rod faces. This wall model allows to take into account the presence of a streamwise pressure gradient and applies both in the viscous sublayer as well as in the inertial region. The reference mesh for the body-fitted $(\mathrm{BF})$ case, $M_{B F}$ contains cells of various sizes, as reported in Tab. 2. $l / h_{\text {rod }}=160$ around the rod, $h_{\text {wake }}$ is the size in the wake region and $h_{f a r}$ is the size elsewhere. The given dimensionless time-step, $\Delta t . u_{r e f} / l$ show the limitation of time discretisation due to the Courant number in this operating condition. The flow-through time is defined as $\tau=l / u_{\text {ref }}$. The spin-up time is $230 \tau$ and $230 \tau$ are also required to gather statistics and perform a frequency analysis.

A visualization of the vorticity field in a plane perpendicular to the rod axis is provided in Fig. 3 (a). The wake of the bluff body presents a coherent vortex shedding where a '2S' mode [40] is observable in the wake. The distribution of time-averaged pressure coefficient , $\left\langle C_{p}\right\rangle$ and its fluctuations on the rod surface are represented in Fig. 4. From the $\left\langle C_{p}\right\rangle$ curve, 


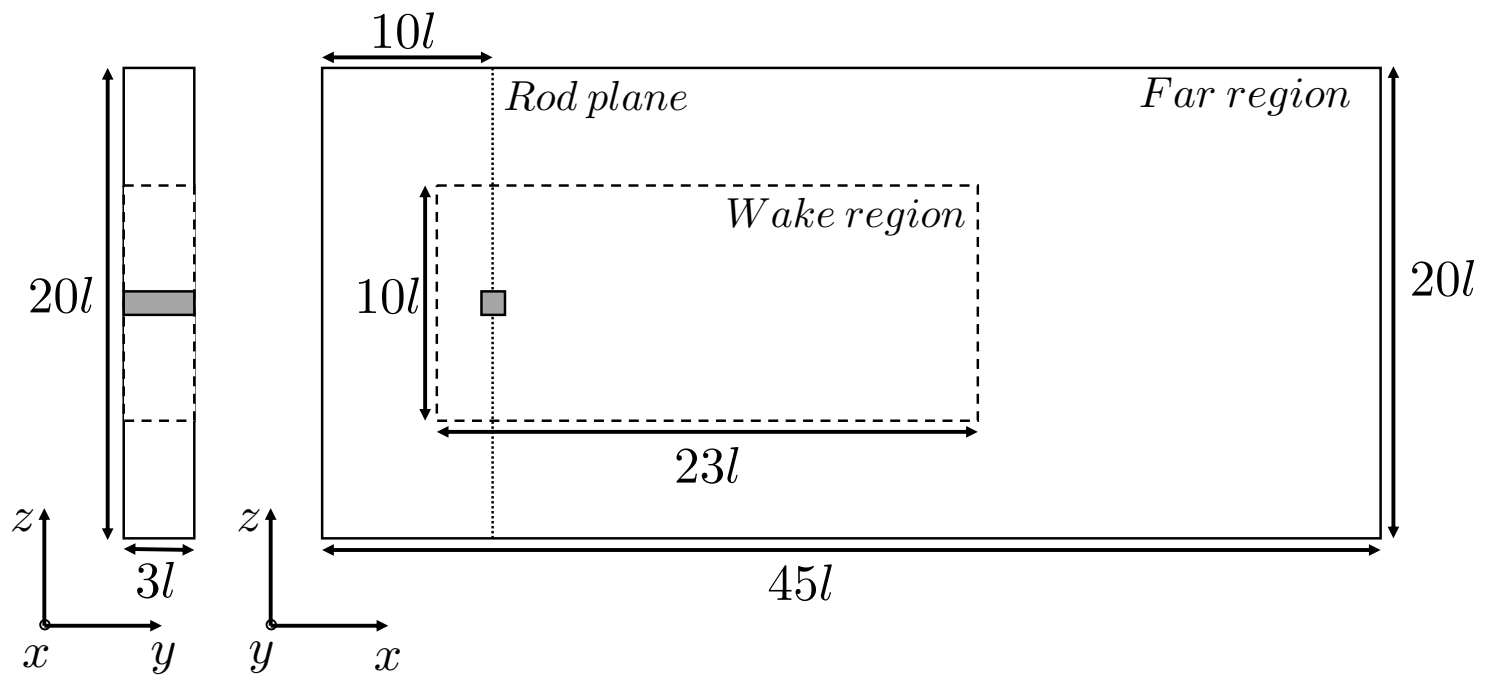

Figure 2: Description of the rod geometry and computation domain. $l$ is the side length of the square rod.

\begin{tabular}{|c|c|c|c|c|c|c|}
\hline Case & $R e\left(\times 10^{4}\right)$ & $\left\langle C_{L}\right\rangle$ & $\left\langle C_{L}^{\prime 2}\right\rangle^{1 / 2}$ & $\left\langle C_{D}\right\rangle$ & $\left\langle C_{D}^{\prime 2}\right\rangle^{1 / 2}$ & $S t$ \\
\hline F. Lesage et al. [35] & 3.3 & - & 1.33 & 2.04 & - & 0.130 \\
C.W. Knisely [36] & $2.2-6.2$ & 0.0 & 1.0 & 2.017 & - & 0.130 \\
B.J. Vickery [37] & $4-16.0$ & - & 1.32 & - & 0.17 & 0.120 \\
\hline LES, reference case & 3.1 & 0.01 & 1.42 & 2.07 & 0.13 & 0.132 \\
\hline
\end{tabular}

Table 1: Aerodynamic coefficients around a square rod obtained from experimental data and from present simulation. The vortex shedding frequency, St, is based on the lift fluctuations.

\begin{tabular}{|c|c|c|c|c|c|c|}
\hline Mesh & $\begin{array}{c}\text { \# elements } \\
\left(\times 10^{6}\right)\end{array}$ & $\begin{array}{c}\text { \# nodes } \\
\left(\times 10^{6}\right)\end{array}$ & $h_{\text {rod }} / l$ & $h_{\text {wake }} / l$ & $h_{\text {far }} / l$ & $\begin{array}{c}\Delta t . u_{\text {ref }} / l \\
\left(\times 10^{-3}\right)\end{array}$ \\
\hline$M_{B F}$ & 344.13 & 88.2 & 0.0063 & 0.0159 & 0.175 & 1.06 \\
$M_{A L M}$ & 3.41 & 0.87 & N/A & 0.0635 & 0.7 & 4.68 \\
\hline
\end{tabular}

Table 2: Mesh characteristics for the rod simulations. 


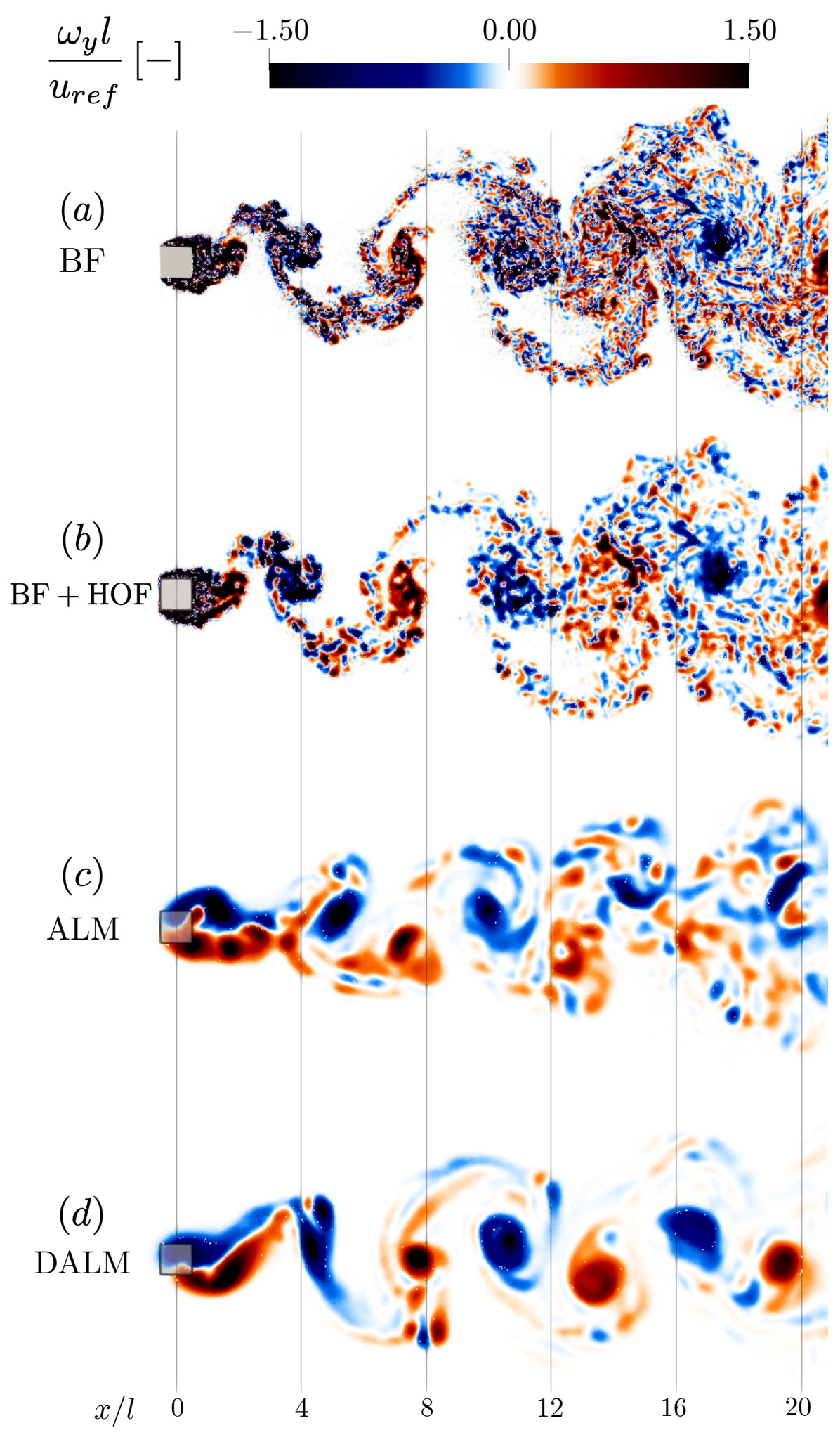

Figure 3: Slice of the instantaneous vorticity magnitude fields in the wake of a square rod for BF (a), filtered BF (b), ALM (c) and DALM (d) cases. 


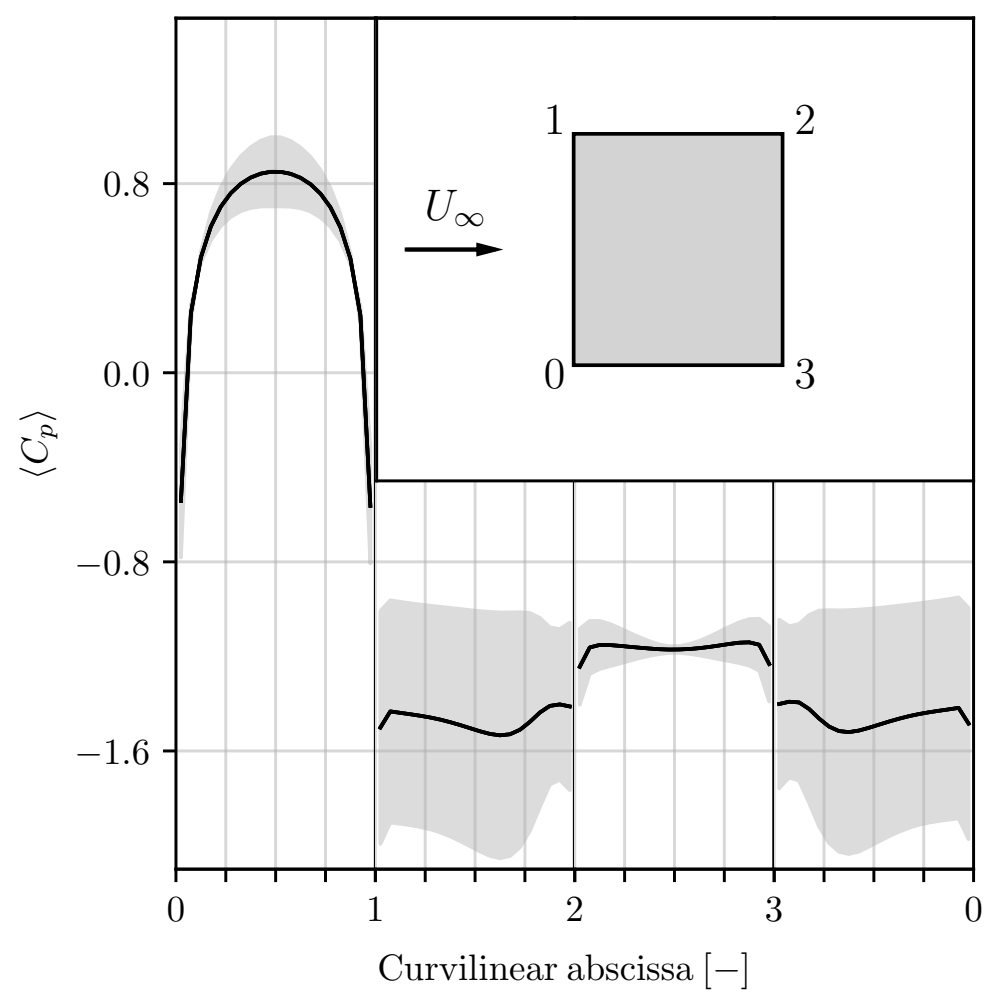

Figure 4: Time and spanwise average of $\left\langle C_{p}\right\rangle(-)$ and its fluctuations $(\square)$ distribution on the rod surface. Front is between vertices 0 and 1 . Top is between vertices 1 and 2. Rear is between vertices 2 and 3 . Bottom is between vertices 3 and 0 .

the drag force is mainly due to the back face of the rod $(2-3)$, with a contribution amounting to $67.4 \%$. The front face induces $32.2 \%$ of the drag and the $0.4 \%$ remaining are due to the friction contribution. The induced lift fluctuations on the top and bottom faces oscillate from $131 \%$ to $-31 \%$. The friction contribution is negligible in comparison to the pressure, which was expected for a bluff body at this Reynolds number as it presents massive boundary layer separation. The computation gives a time-averaged dimensionless wall distance $\left\langle y^{+}\right\rangle$around 10 which is above the limit of the linear viscous sub-layer. The use of the wall model of Duprat et al. [39] is well suited for this near wall resolution.

As the aim of the proposed technique is to generate realistic turbulent structures downstream of a turbulence grid, the proper modeling of the shear stress at the rod walls is of less importance. Integrating the instantaneous pressure and friction coefficient allow obtaining the resulting lift and drag coefficient of the rod. Their fluctuations are presented in Fig. 5, with their corresponding spectrum. Table 1 presents the mean, the root-mean-square and the Strouhal number that are computed from these signals. These values are within the range of the available experimental data.

\subsection{Assessment of the standard ALM method}

The ability of the ALM to reproduce rod wakes at a low computational cost is assessed here. As it seems natural to model the flow around an airfoil using an ALM, it is less obvious that this method is suitable for bluff-body flows that feature massive boundary layer separation with vortex shedding.

The assessment of the method is performed using a continuous mesh (i.e. that does not 

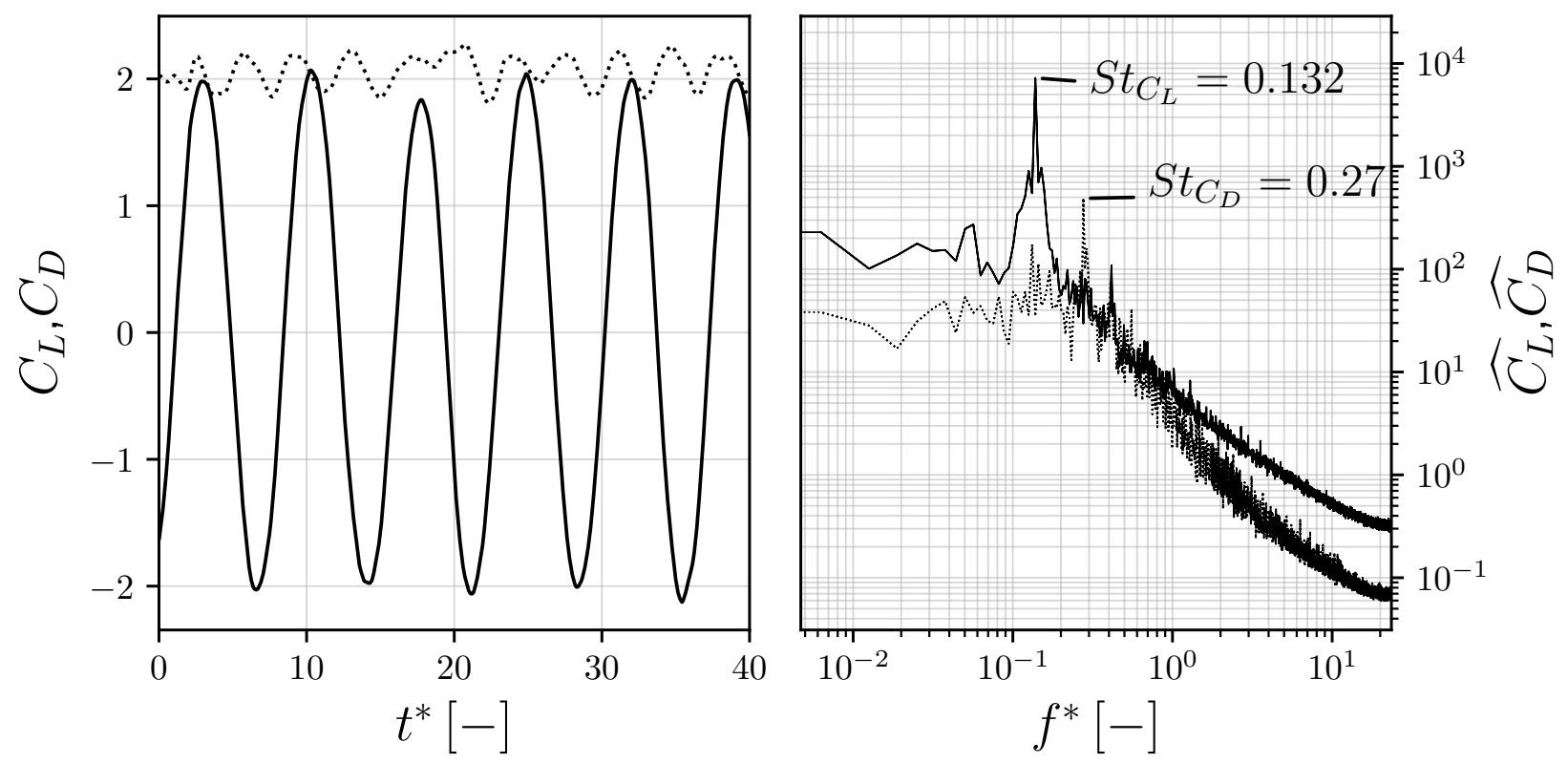

Figure 5: (a) Evolution of Lift, $C_{L}(-)$, and Drag, $C_{D}(\cdots \cdots)$, coefficients over dimensionless time for the wake of a 3D square rod at $R e=3 \times 10^{4}$. (b) Spectrum of both signals are presented as $\widehat{C_{L}}(-)$, and $\widehat{C_{D}}(\cdots \cdots)$.

include a solid body representing the rod) referred here as $M_{A L M}$. The characteristics of this mesh are summarized in Tab. 2. The rod is discretized along its span with 15 actuator elements in order to obtain $w / l=\epsilon / l=0.2$. The influence of the rod on the flow is taken into account by the ALM using as input the mean aerodynamic coefficients, $\left\langle C_{L}\right\rangle$ and $\left\langle C_{D}\right\rangle$ from the reference case summarized in Tab. 1 .

The flow topology provided by the ALM is displayed in Fig. 3 (c). The ALM method generates coherent vortical structures that are shed in the wake. For $x / l>12$, the wake has lost its coherence while in the BF case, coherent structures can be identified in the far wake. The small scale content of the ALM turbulent wake is also lower: this was expected as the mesh of the reference case (BF) is four times finer compared to that of the ALM grid.

In order to make a fair comparison between both methods, the instantaneous results of the reference case (BF) are filtered using a high order filter (HOF) (10 ${ }^{\text {th }}$-order) 41,42 such that its filter size is equal to the ALM mesh size, see Fig. 3 (b).

Even though the small scale content of the filtered BF vorticity field is still higher compared to the ALM solution, the filtering allows identifying the topology of the large scale vortical filaments.

Flow statistics are provided in Fig. 6. The velocity profiles are extracted at five positions downstream of the rod. Even if the flow topology provided by the ALM seems correct on a qualitative basis, the mean and fluctuating velocity profiles feature discrepancies compared to the reference case. Indeed, velocity fluctuations profiles are significantly smaller in the center of the wake and exhibit a flatter profile compared to the reference case.

\subsection{Description of the Dynamic Actuator Line Method (DALM)}

As the standard ALM method features modest performance to reproduce turbulence fluctuations, a variant of this method is proposed here. A first assessment of this new method is 

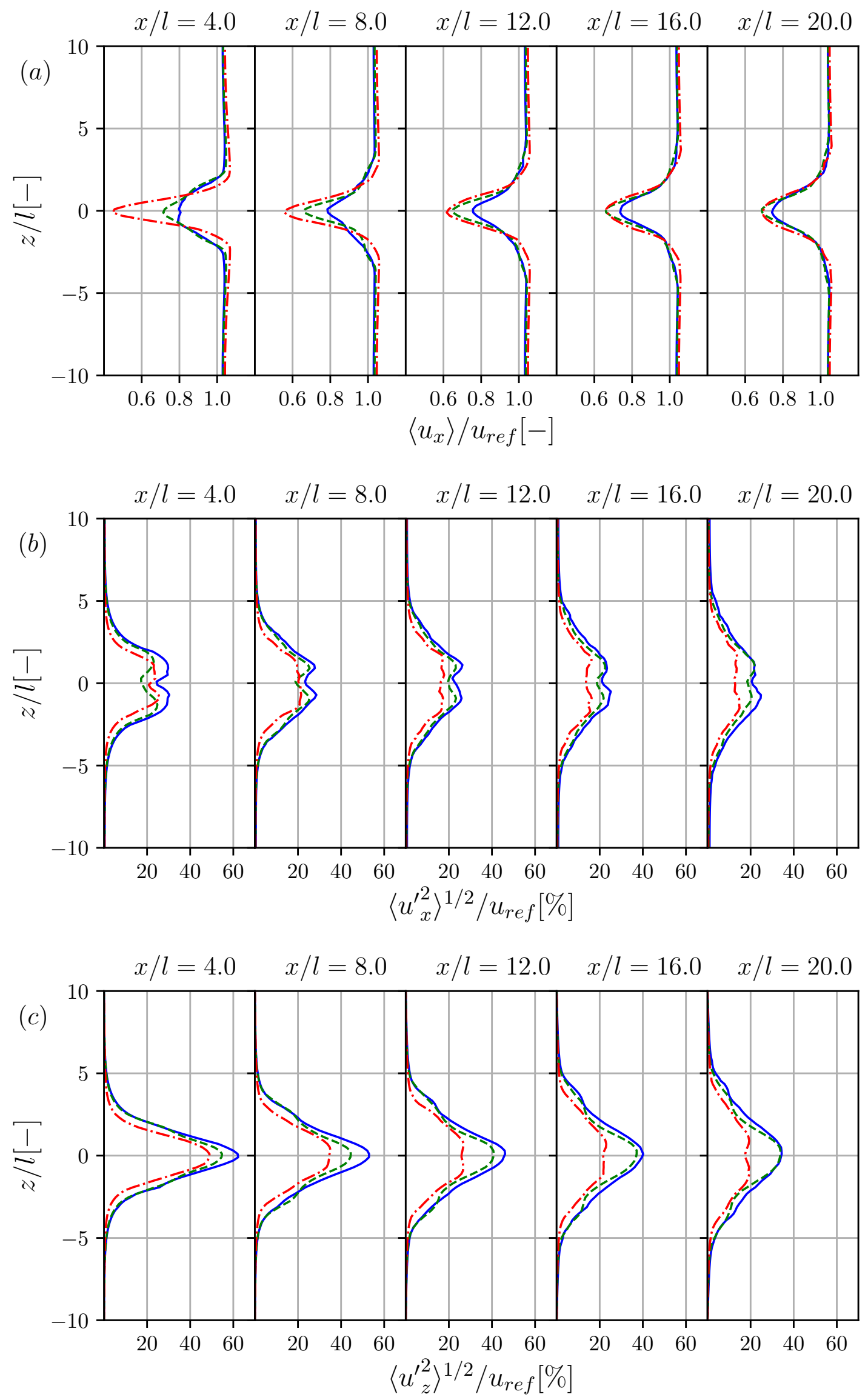

Figure 6: Mean streamwise velocity (a), RMS of streamwise velocity component (b) and RMS of vertical velocity component (c) behind the rod at $x / l=4,8,12,16$ and 20 for BF (-), ALM (--.) and DALM (-- -). 


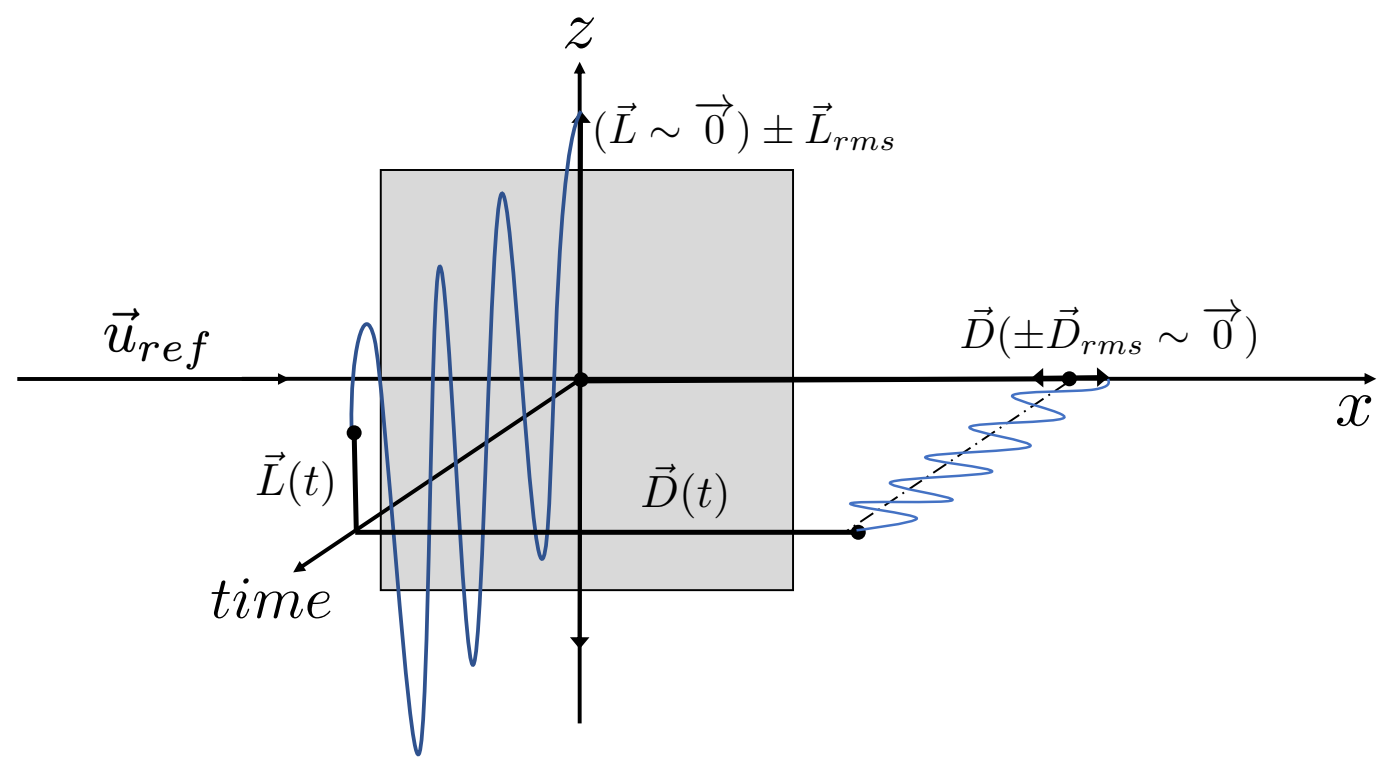

Figure 7: Oscillating lift and drag forces evolution over time for one section of a rod.

performed on the single square rod test-case presented above. It is shown here that it is required to take lift fluctuations into account in order to provide a correct modeling of the bluff body wake fluctuations.

The lift coefficient variations is superimposed to the lift coefficient of the ALM formulation $\left\langle C_{L}\right\rangle$ as pictured in Fig. 7 The time variation of the coefficients is thus written as: $\mathrm{C}_{L}(t)=\left\langle C_{L}\right\rangle+\left\langle C_{L}^{\prime 2}\right\rangle^{1 / 2} \sqrt{2} \sin (2 \pi f t+\tilde{\varphi})$,

$C_{D}(t)=\left\langle C_{D}\right\rangle$, where $f$ is the frequency of the vortex shedding and $\tilde{\varphi}$, the random phase of the fluctuations that has to be modeled. The aim of this random phase is to de-synchronize the vortex shedding when more than one rod is taken into account. The drag fluctuations are not taken into account here since they are small. As a consequence, they will not affect the largest structures of the wake (this point is further discussed in section 3.4).

Even though this new Actuator Line Method requires to set the RMS of the lift and the Strouhal number, it remains easy to implement. Theses physical properties can be retrieved, for wide variety of static or translating bluff bodies from the literature [43 45]. In the next section, this new formulation will be applied and compared in the case of a square rod wake. An application to grid turbulence generation will be provided in section 4 .

\subsection{Validation of the DALM}

The validation of DALM formulation is performed on the flow around a square rod. The results are compared with those of the reference body fitted computation and classical ALM. The mesh $M_{A L M}$ and the spanwise discretization of the rod are the same as in the ALM case. The DALM result presents a vortex shedding frequency that is similar to the reference case (see Fig. 3). As the boundary layers are not captured with the DALM, small scale vortices are not present in the wake. Nevertheless, the large scale coherent structures generated at the rod location are very similar to those obtained with the BF case and they are also transported similarly.

Flow statistics are compared using the mean streamwise velocity in the wake (Fig. 6(a)) and the RMS values of vertical and streamwise velocity components (Fig. 6(b) - 6(c)) for profiles 
at five positions downstream of the rod. Considering the mean wake deficit, all the profiles are almost coincident for $x / l>20$. The velocity fluctuations profiles (vertical and streamwise) obtained using the DALM have the same shape as the BF case, two local maxima for the streamwise component and one single maximum for the vertical fluctuations.

A more detailed analysis of the velocity fluctuations is provided through a frequency analysis, depicted in Fig. 8. The velocity signals are gathered over $230 \tau \approx 1.08 \mathrm{~s}$ with a fixed timestep $d t=0.1 \mathrm{~ms}$ at $x / l=4,8,12,16$ and 20 for the three cases. The normalized 1D transverse energy spectra $E_{z z}$ are provided in Fig. 8. The vertical doted lines are located on the eigenfrequency of the reference case: $f_{0}^{*}=0.137$, and its second harmonic $f_{2}^{*}=0.273$. The same eigen-frequency can be retrieved for the DALM case showing a good behavior of the wake fluctuations. This is not the case for the "standard" ALM case with a higher eigen-frequency $f^{*}=0.169$. Furthermore, the relative amplitudes at the eigen-frequencies are very similar for both the reference and DALM cases, while velocity fluctuations provided by ALM decay rapidly in the wake. Another important point is the difference of cut-off frequency between the reference case and the actuator line cases, a direct result of the mesh resolution.

The cut-off frequency of the BF mesh is such that $f_{\text {cut off,BF }}^{*} \approx 8$. The two other cases have a four times smaller frequency cutoff: $f_{\text {cut off,ALM }}^{*} \approx 2$. In this study, it doesn't impact the frequency range of interest.

It has been shown in this section that DALM was able to improve the description of the flow behind a single square rod, compared to the classical ALM. The aim of the next section is to apply this new method to the simulation of a turbulence generation grid.

\section{Application to a turbulence generation passive grid}

The DALM proposed here is applied to the turbulence generation system of the "NTNU Blind Test 5" [46] wind tunnel. Several modeling strategies are compared to experimental measurements: i) DALM, ii) resolved flow around the rods (Body Fitted, BF), and iii) Homogeneous Isotropic Turbulence (HIT) injection.

\subsection{Case description}

The wind tunnel dimensions are $L_{x} \times L_{y} \times L_{z}=14 \times 2.71 \times 1.801 \mathrm{~m}^{3}$, see Fig. 9. The turbulence grid, located at $x_{0}$, is composed of $l \times l=47 \times 47 \mathrm{~mm}^{2}$ square cylinder rods [47]. The grid presents a constant horizontal spacing between the vertical rods of 5.1l. The horizontal rods are arranged with an increasing vertical spacing from the floor to the roof [47]. The purpose of this arrangement is to generate a vertically sheared flow profile after the grid. The horizontal rods are upstream the vertical rods and both are bind to each other at each grid nodes, i.e. horizontal and vertical rods section center are shifted by $l$ in the streamwise direction. The resulting grid blockage ratio, $\sigma$, is $34.5 \%$ in this wind tunnel and the effective grid mesh size [6] defined as $M_{\text {eff }}=\frac{4 T^{2}}{P} \sqrt{1-\sigma}$ is $M_{\text {eff }}=8.5 l$, where $P$ is the perimeter of the grid and $T^{2}=L_{y} \times L_{z}$. Experiments have shown that upstream of the grid, the streamwise velocity profile is flat with $u_{x}=u_{r e f}=10 \mathrm{~m} \cdot \mathrm{s}^{-1}$ while downstream, this profile can be accurately described by a power law:

$$
\frac{u_{x}(z)}{u_{r e f}}=\left(\frac{z}{L_{z}}\right)^{\alpha},
$$

where $z$ is the height in the wind tunnel and the power coefficient $\alpha=0.11$ is selected to fit experimental data [47]. Experiments provide velocity and turbulent intensity profiles at three 


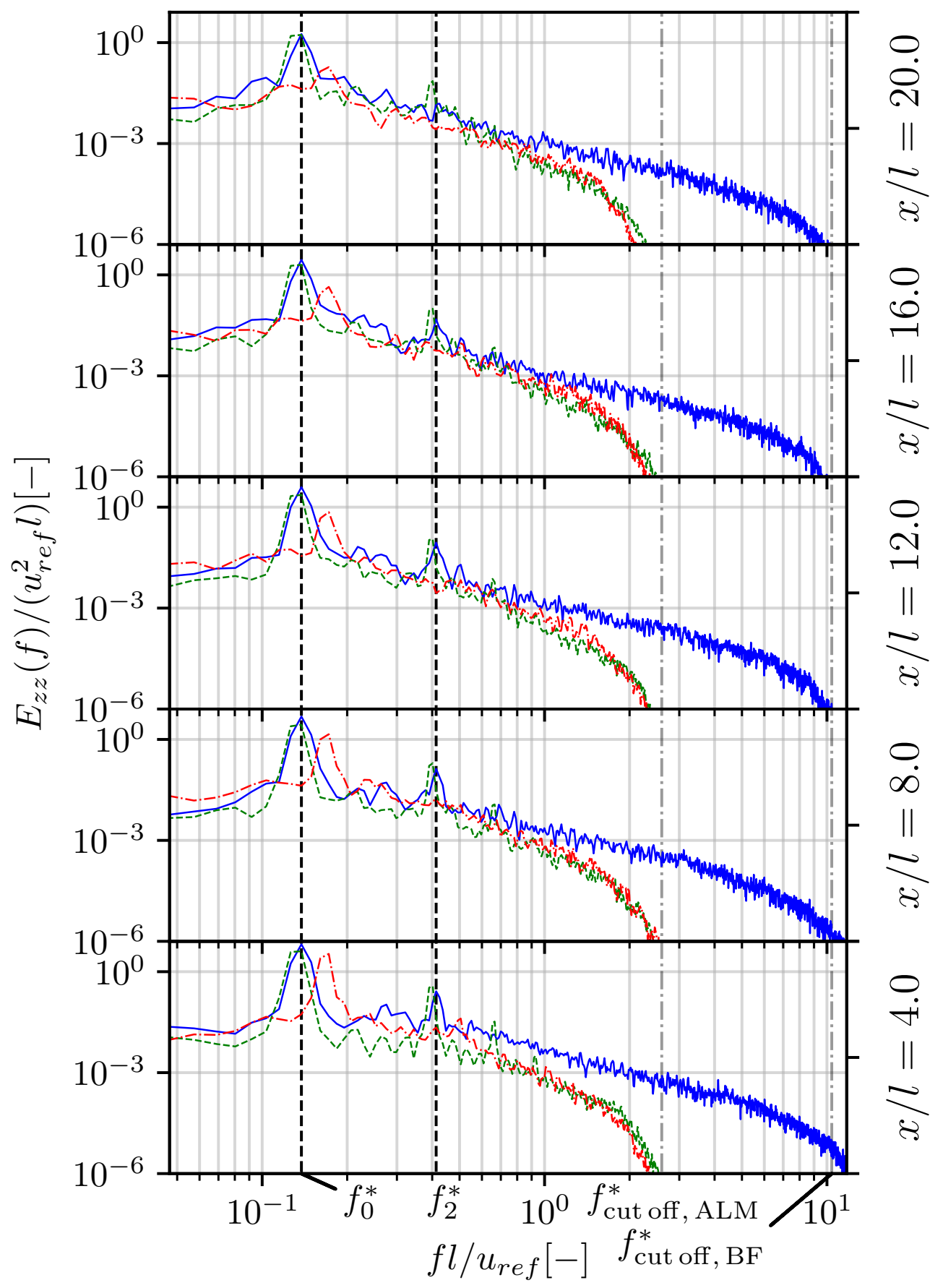

Figure 8: 1D Energy spectra of the vertical velocity behind the rod at at various axial location, with $f^{*}=f l / u_{r e f}$, for BF (-), ALM (--) and DALM (-- ). 


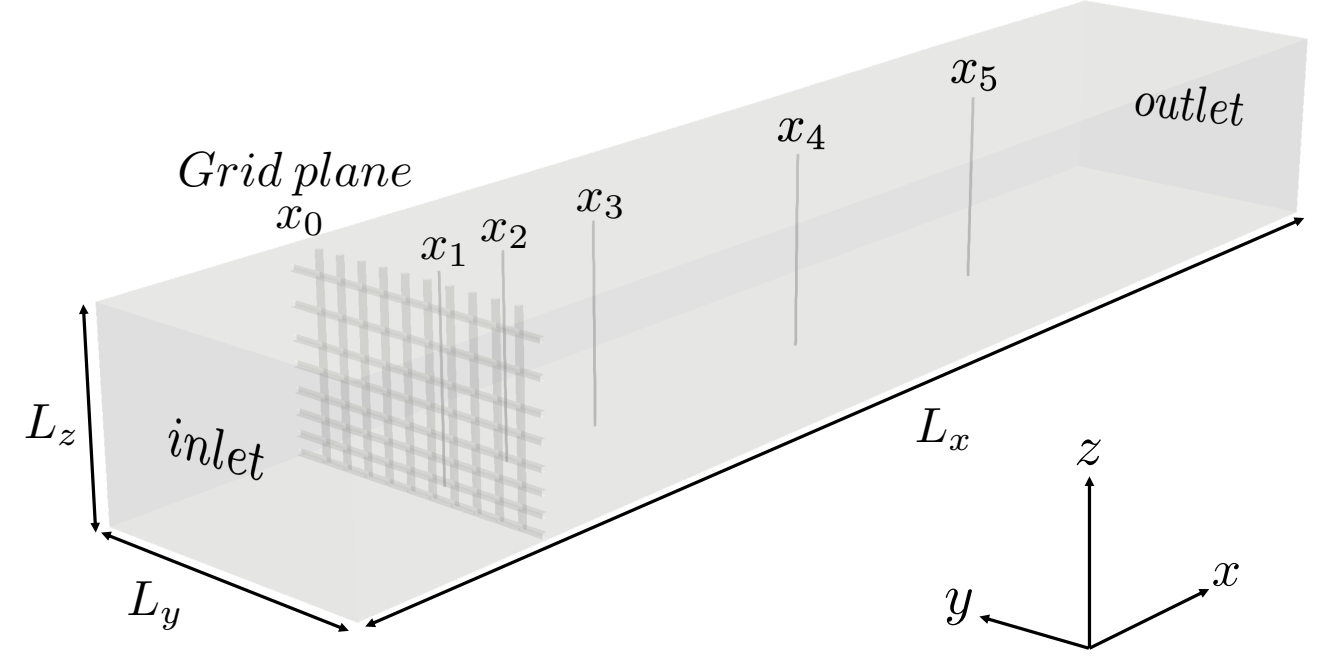

Figure 9: Visualization of the computation domain of NTNU BT5 with grid only. The downstream grey vertical lines $\left(x_{3}, x_{4}\right.$ and $\left.x_{5}\right)$ refer to experimental measurements.

positions downstream the grid $x_{3} / l=38, x_{4} / l=95$ and $x_{5} / l=152$, presented as grey lines in Fig. 9. Although not present in the experimental data-set, the positions $x_{1} / l=5$ and $x_{2} / l=20$ are also investigated numerically to study the grid wake destabilization.

For all cases, the flow-through time is taken according to the distance between $x_{0}$ and $x_{5}$ and equal to $\tau=\left(x_{5}-x_{0}\right) / u_{r e f}=0.715 \mathrm{~s}$. The physical duration of the three simulations is $7 \tau$ for the flow establishment and $15 \tau$ for statistics accumulation.

\subsection{Computational setup}

The aim of this work is to assess the DALM and compare it to other strategies: a resolved flow around the rods, set as reference and a Homogeneous Isotropic Turbulence (HIT) injection with experimental velocity profile. Each of these methods requires different mesh resolutions: for the $\mathrm{BF}$ case, the mesh size around the rods needs to be fine enough to capture the boundary layer. This significantly increases the number of elements in the grid with a total of around $284 \times 10^{6}$ tetrahedra for BF case. The two other methods do not require such a fine mesh and contains approximately $157 \times 10^{6}$ elements for DALM case and $139 \times 10^{6}$ elements for HIT case. Theses meshes are illustrated in Fig. 10. A wall model [39] is applied on the wind tunnel walls. Although the three cases are very similar in most aspects, the peculiarities of each approach are presented in the following sections.

\subsubsection{DALM case}

In this case, a flat velocity profile, upstream of the grid, is set at the inlet of the computational domain with $u_{x}=10 \mathrm{~m} . \mathrm{s}^{-1}$. The grid resolution near the position of the rods is the same as in the wake region, $h / l \approx 0.08$ as shown in Fig. 10 . The rods are discretized with DALM in order to obtain $w / l=\epsilon / l=0.2$. Therefore, according to the turbulence grid geometric properties the horizontal and vertical rods are composed with 288 and 190 actuator elements, respectively. In order to apply the DALM presented above, the aerodynamic coefficients of the square rods are needed. Moreover, those rods are not isolated anymore and those coefficients need to take into account the rods interactions, between each other but also with the wind tunnel walls. Literature provides experimental data for two parallel rods interacting at different distances [48] or a single rod interacting with a wall [49. Taking those effects into account is mandatory to recover the correct mean velocity shear. Here, the interactions between crossing vertical and 


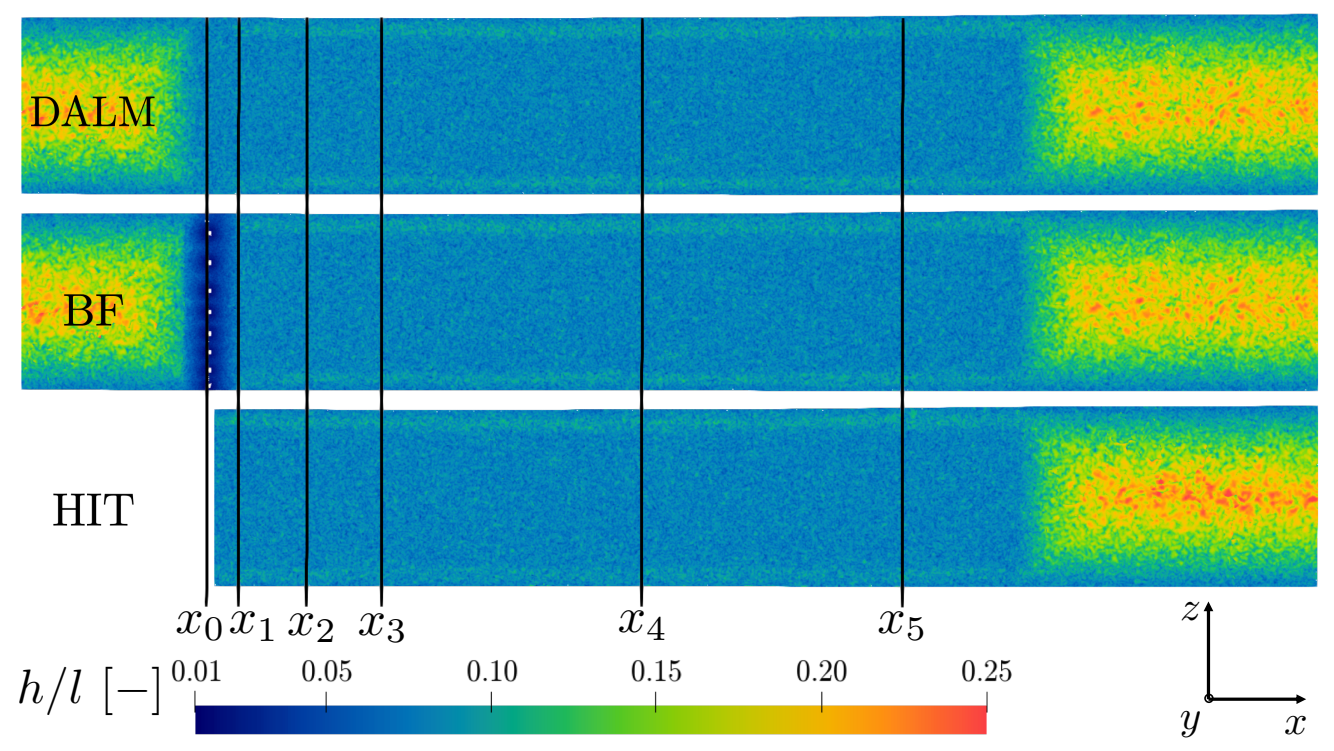

Figure 10: Side view of the unstructured grid size, $h / l$, used to capture grid turbulence generation. From top to bottom: DALM, BF and HIT mesh. The mesh size $h$ is defined here as the cubic root of the cell volume. It can be seen that $h / l \approx 0.08$ in the wake region for the three cases.

\begin{tabular}{|c|c|c|c|c|}
\hline Rod & $\left\langle C_{L}\right\rangle$ & $\left\langle C_{L}^{\prime 2}\right\rangle^{1 / 2}$ & $\left\langle C_{D}\right\rangle$ & $S t$ \\
\hline Vertical, 1-10 & 0.0 & 1.21 & 2.16 & 0.128 \\
Horizontal, 1 & -0.154 & 1.34 & 2.22 & 0.128 \\
Horizontal, 2 & 0.00 & 1.39 & 2.23 & 0.128 \\
Horizontal, 3 & 0.00 & 1.34 & 2.21 & 0.128 \\
Horizontal, 4 & 0.00 & 1.29 & 2.19 & 0.128 \\
Horizontal, 5 & 0.00 & 1.24 & 2.17 & 0.128 \\
Horizontal, 6 & 0.00 & 1.20 & 2.14 & 0.128 \\
Horizontal, 7 & 0.00 & 1.16 & 2.11 & 0.128 \\
Horizontal, 8 & 0.00 & 1.16 & 2.11 & 0.128 \\
\hline
\end{tabular}

Table 3: Aerodynamic coefficients of the grid rods depending on their environment [48,49].

horizontal rods are neglected. Moreover, no specific treatment have been applied on the mollification process at theses locations: vertical and horizontal rod mollification kernels overlap. This assumption relies mainly on the observation that these crossings marginally contribute to the blockage ratio. The spacing of the vertical bars remains constant and the aerodynamic coefficients based on the rod/rod spacing are used. As for the horizontal bars, the rod/rod spacing and wall/rod spacing are used to evaluate the aerodynamic coefficients [48,49]. The resulting coefficients are summarized in Table 3. It can be observed that the drag coefficient and the lift fluctuations decrease as the spacing between rods gets larger. Experimental studies [48,49] have shown that the Strouhal number is not impacted by such high spacing. For the first horizontal rod, the negative mean lift is due to the wall proximity which is lower than 0.4l. An other assumption lies in the rod to rod wake synchronization through the phase offset $\tilde{\varphi}$ of DALM process. This parameter has been investigated by Chatterjee and Biswas [50] for a laminar flow passing through a homogeneous grid. Due to the flow complexity and the grid geometry, the phase of each rod is here set randomly at the computation initialization. This prevents the creation of large-scale coherent structures that would be convected in the wind tunnel. 


\subsubsection{BF case}

This case uses a "brute force" approach in which the grid influence is resolved similarly to section 3. As in DALM case, the inlet velocity profile is set as a flat velocity profile, upstream of the grid. The mesh takes into account the rod geometry with a cell size around the grid rods of $h_{\text {rod }} / l \approx 0.01$. This leads to $\left\langle y^{+}\right\rangle<50$. Consequently, the first mesh point is not in the viscous sub-layer and a wall model must be used: Duprat et al. [39] wall model is chosen here.

\subsubsection{HIT case}

In this case, a precursor is used to simulate the flow past the grid without considering the rods. Therefore, the inlet boundary of the domain is located at $x=2 l$ (Fig. 10). The inlet velocity flow profile is set according to experimental data with a fitted power law (see Eq. 9). A Passot-Pouquet [51] spectrum is used to generate the homogeneous isotropic turbulence. In order to represent the grid generated turbulence using HIT injection, it is required to estimate the turbulence intensity and the integral length scale at the inlet. The integral length scale is chosen to account for the grid constant spacing, $l_{t}=5.1 l$. The estimation of the streamwise turbulence intensity, defined as $T I=\left\langle u_{x}^{\prime 2}\right\rangle^{1 / 2} / u_{\text {ref }}$, requires experimental results and therefore its value downstream of the grid. The Frenkiel-Roach correlation established for a square rod grid with homogeneous spacing [5] is used for this purpose:

$$
T I=c\left(\frac{x}{l}\right)^{-n}
$$

where $c$ is a constant depending on the grid geometry $(c=1.13$ when the grid spacing is homogeneous [5]), $n$ a constant evaluated at 5/7 experimentally, $x$ the distance to the grid and $l$ the rod width.

The turbulence intensity was measured experimentally at three positions downstream of the grid. With this data, the evaluated TI from Eq. 10 is $65 \%$. This value is really high and, as expected after a first simulation, overestimates the experimental turbulence intensity profiles.

In order to obtain better results with the HIT injection method, a "trial and error" strategy was used: five different $T I$ values were tested to match experimental results. The final chosen $T I$ value is $35 \%$. In what follows, the flow fields presented for homogeneous isotropic turbulence injection were obtained with $T I=0.35$.

\subsection{Flow visualization}

Figure 11 shows the mid vertical plane colored by the instantaneous norm of vorticity. This allows to observe how the vorticity decays along the wind tunnel. The rods wakes destabilization just downstream $x_{1}$ position can be observed for both DALM and BF cases. Therewith, two regions appear downstream of the grid, the mixing region and the decay region. In the mixing region, the wake of the grid is not destabilized, involving coherent flow structures. Indeed, the wakes interact with each other to generate a fully turbulent flow between $x_{1}$ and $x_{2}$, depending on the grid vertical spacing. Further downstream, in the decay region, turbulence becomes homogeneous and its intensity decays along the wind tunnel. This phenomenon is not observed for the HIT case since the injected homogeneous isotropic turbulence is already fully developed and the actual grid geometry is not represented.

\subsection{Wake destabilization}

It has been shown that the proper capture of the wake destabilization is highly dependent of the mesh resolution [52,53]. The importance of this phenomena on the flow statistics is investigated in this subsection. 


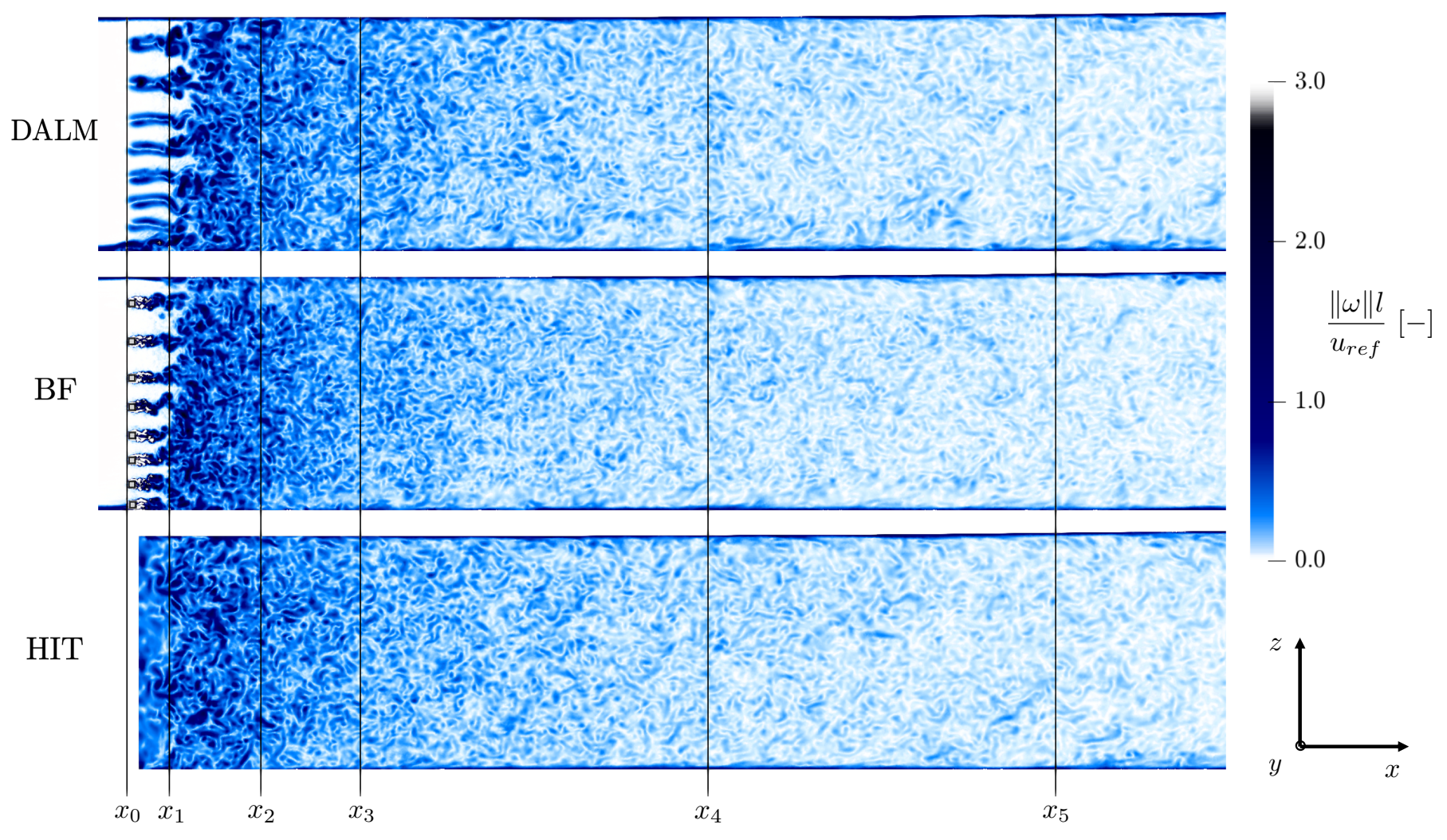

Figure 11: Magnitude of the vorticity field in the vertical plane of the wind tunnel for all cases. From top to bottom: DALM, BF and HIT.

\subsubsection{Mixing and decay regions}

Figure 12 shows the streamwise turbulence intensity as a function of the downstream position at two different lateral positions, one behind a vertical rod of the grid and the other between two rods. For the reference BF and DALM cases, the two TI curves cross at $x \approx 10 l$ and show a significant decay after this position, in agreement with the Frenkiel-Roach equation. Nevertheless, discrepancies can be observed on the maximum values of the TI between theses cases. For the HIT injection case, the two curves are similar since the mixing region can not be reproduced by this method. The behavior of all cases is similar beyond $x_{2}$ and matches the experimental turbulence intensity value in $x_{3}$.

The quantification of the turbulence anisotropy is investigated inside the mixing and decay regions. The anisotropy invariants [54] are computed using the eigenvalues of the Reynolds stress anisotropy tensor:

$$
a_{i j}=\frac{\left\langle u_{i}^{\prime} u_{j}^{\prime}\right\rangle}{2 k}-\frac{\delta_{i j}}{3}, \text { wherek }=\sum_{i=1}^{3} \frac{\left\langle u_{i}^{\prime} u_{i}^{\prime}\right\rangle}{2} .
$$

Using the barycentric map developed by Banerjee et al. [55], any anisotropy tensor can be associated to a point inside an equilateral triangle, whose vertices correspond to three limiting states of turbulence:

- $\mathbf{x}_{1 c}$ : one-component or "cigar-shaped" turbulence

- $\mathbf{x}_{2 c}$ : two-component or "pancake-like" turbulence

- $\mathbf{x}_{3 c}$ : isotropic or spherical turbulence 


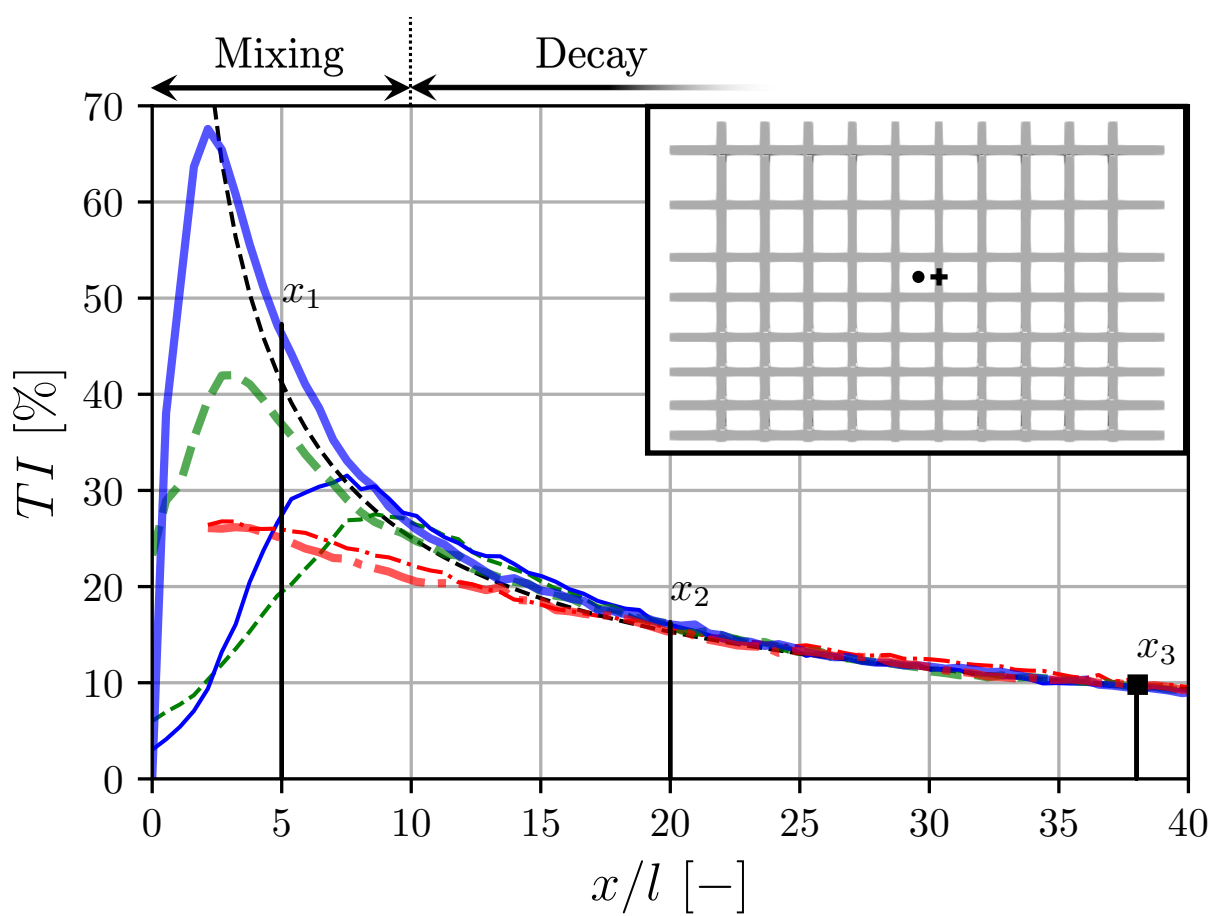

Figure 12: Streamwise evolution of streamwise turbulence intensity, $T I$, at two different lateral locations on the turbulence grid for the three cases. One at the center of the grid mesh, $y / l=0$ (thin line), and the other behind a rod of the grid, $y / l=2.55$ (thick line). DALM (- -), BF (一), HIT (--.), experiment at $x_{3}(-)$ and Eq. $10(--)$.
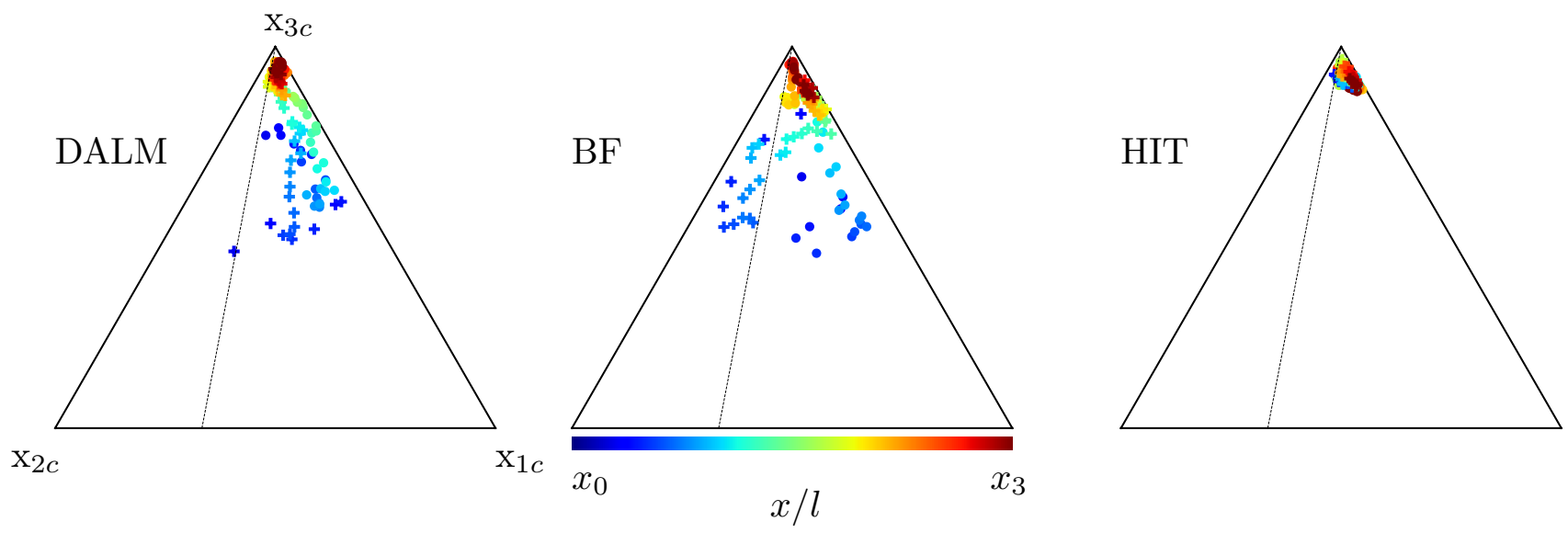

Figure 13: Barycentric maps showing the streamwise evolution of the anisotropy invariants behind the grid, color depends on the position between $x_{0}$ and $x_{3}$. Two positions on the grid are presented, one at the center of the grid mesh, $y / l=0(\bullet)$, and the other behind a rod of the grid, $y / l=2.55(+)$, see Fig 12 . The three different cases, A, B and C are presented. The limiting states of componentality are labeled along with the plane-strain limit (.....). 
Let $\lambda_{1}, \lambda_{2}$ and $\lambda_{3}$ be the eigenvalues of a given anisotropy tensor: this tensor is represented inside the above described triangle by the point $\mathbf{x}_{B}$ such that:

$$
\mathbf{x}_{B}=\sum_{i=1}^{3} C_{i c} \mathbf{x}_{i c},
$$

where the weights are computed as

$$
\begin{aligned}
& C_{1 c}=\lambda_{1}-\lambda_{2}, \\
& C_{2 c}=2\left(\lambda_{2}-\lambda_{3}\right), \\
& C_{3 c}=3 \lambda_{3}+1 .
\end{aligned}
$$

By definition, these weights sum to one. Figure 13 presents the barycentric map of the DALM, $\mathrm{BF}$ and HIT cases. The invariants are computed along the two lines presented on Fig. 12 , i.e. in the wake of a rod and at the center of a grid cell. A major observation from the barycentric map is that all methods present an isotropic state of turbulence in the decay region. The turbulence state of the HIT case is always isotropic since the injected velocity fluctuations represent a developed isotropic state. Discrepancies in the mixing region can be observed between $\mathrm{BF}$ and DALM case. Indeed in the wake of the grid rod, the actuator grid doesn't reproduce the effective state of turbulence. This shows one limit of the method to emulate the wake of a turbulence grid. A better estimation of the mixing region turbulence state could be provided by improving the phasing strategy for the vortex shedding in DALM.

From these observations, the destabilization of the grid wake remains an important phenomena in order to generate an homogeneous and isotropic turbulence in the flow.

\subsubsection{Time-averaged velocity and turbulence intensity profiles}

The flow statistics are compared considering the profiles of mean streamwise velocity, Fig. 14(a), and streamwise turbulence intensity, Fig. 14(b), at five positions downstream of the grid. A comparison with the experimental profiles is provided for positions in the decay region, at $x_{3}$, $x_{4}$ and $x_{5}$.

In the mixing region, at $x_{1}$, the BF and DALM cases are able to represent the rod wakes through a velocity deficit at the position of the rods, while the sheared profile cannot be observed. They present thus a similar profile shape with an underestimated amplitude for the DALM case. The HIT injection presents a sheared profile, which is expected since the experimental profile is used as an input parameter. Right after the destabilization, all cases present a sheared velocity profiles shape. Still, the reference and DALM cases present fluctuations due to the velocity deficit behind the grid rods with a slight over-prediction of the DALM. The destabilization of the grid wake is important here, as mentioned in subsection 4.4.1. The timeaveraged streamwise velocity profiles of all the presented cases are in good accordance with the experimental results in the decay region. The BF and DALM cases both show a fair prediction of the downstream velocity field.

The streamwise turbulence intensity profiles (Fig. 14(b)) are also investigated. In the mixing region, between $x_{0}$ and $x_{2}$, the reference case and DALM present high levels of fluctuations, up to $35 \%$ at $x_{1}$. The profiles become nearly flat right after the destabilization of the wake and nearly homogeneous in the middle of the wind tunnel. The experimental data are only available after $x_{3}$ but at this position, the profiles of all the cases are already identical. 

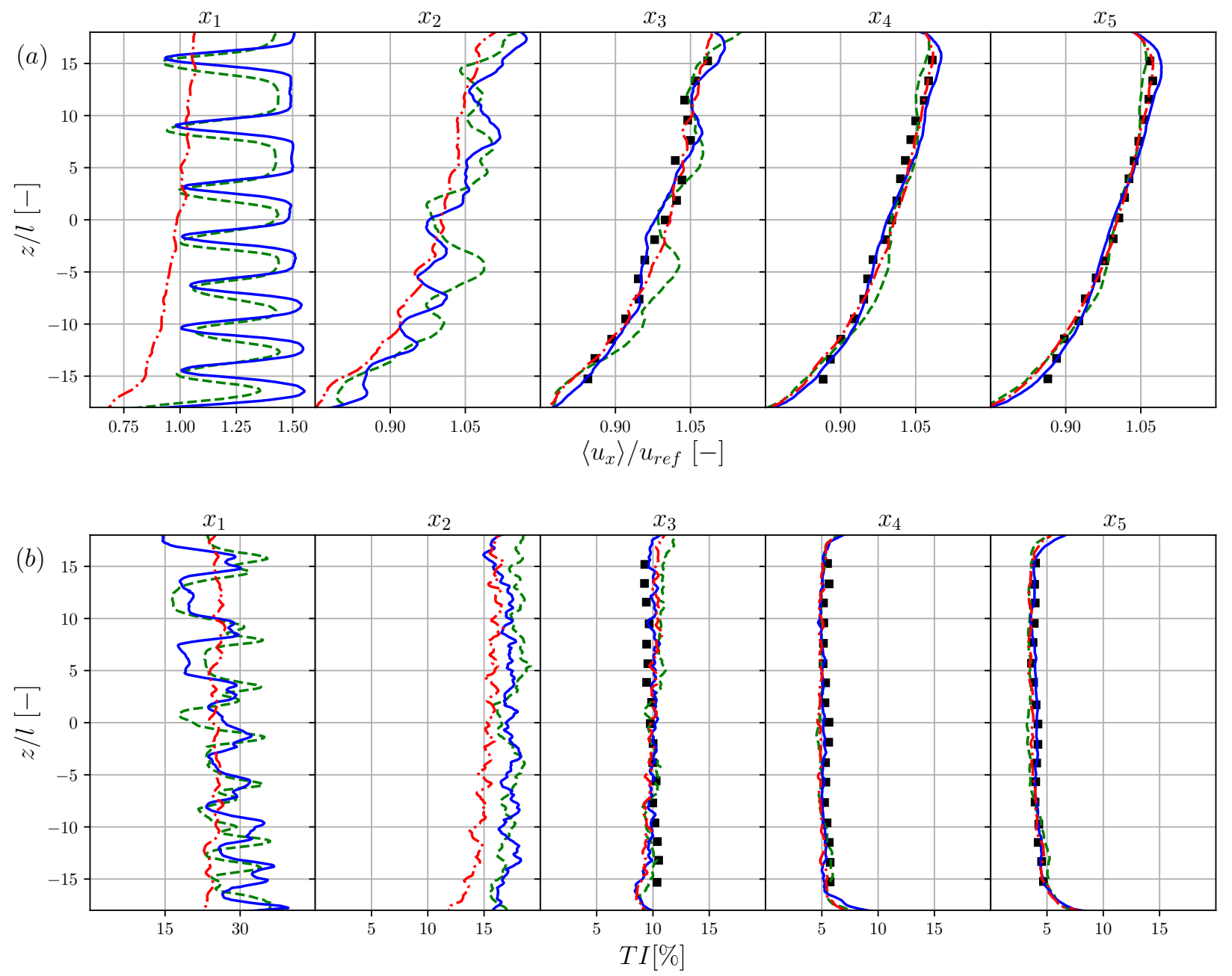

Figure 14: Vertical profiles of mean streamwise velocity (a) and turbulence intensity (b) at $x_{1}, x_{2}, x_{3}, x_{4}$ and $x_{5}$ : experiment (-), DALM (-- ), BF (-) and HIT (-..). 

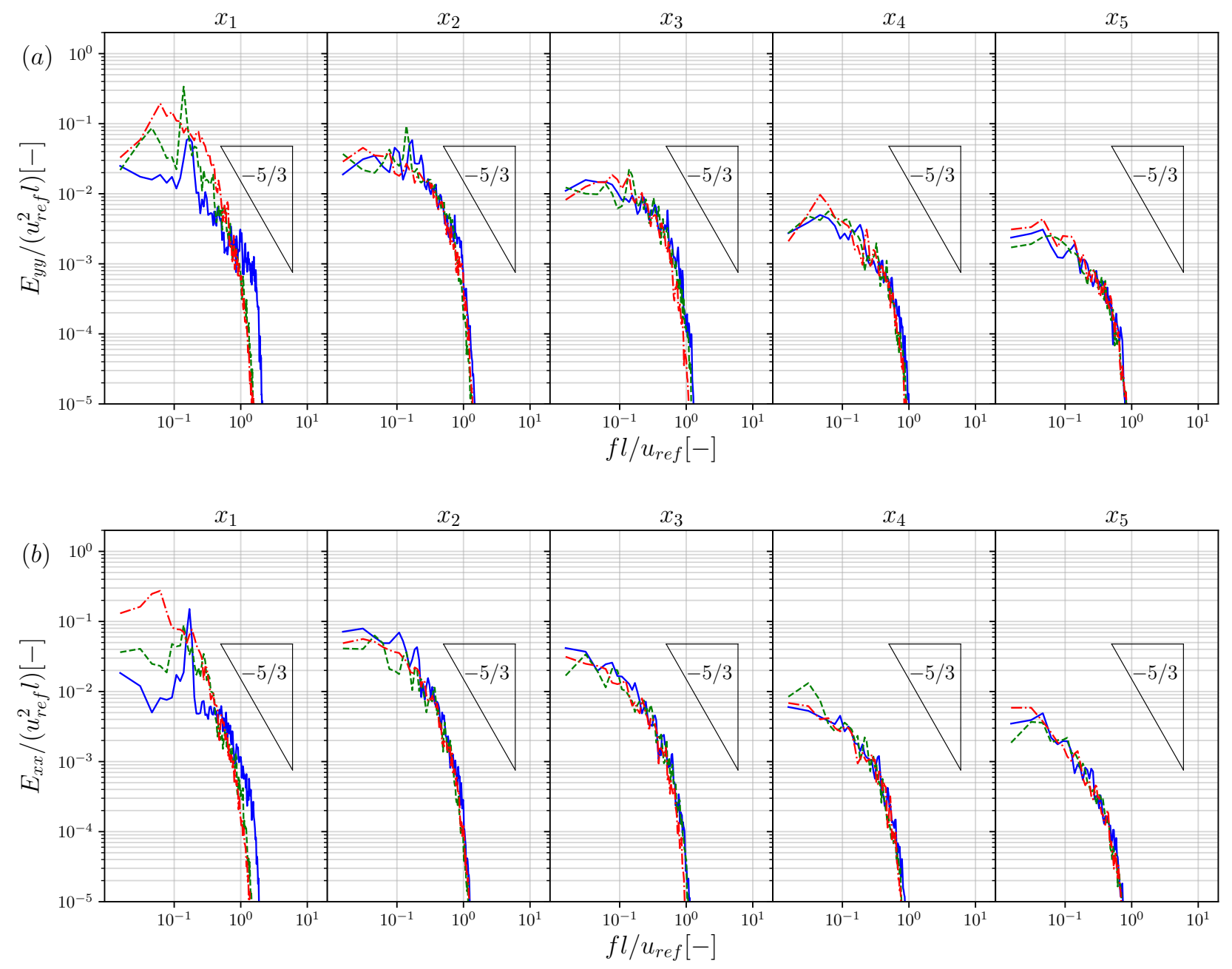

Figure 15: 1D power spectra of the transverse velocity fluctuations (a), $E_{y y}$, and the streamwise velocity fluctuations (b), $E_{x x}$ behind the rod at stations $x_{1}, x_{2}, x_{3}, x_{4}$ and $x_{5}$ for DALM (-- ), $\mathrm{BF}(-)$ and $\operatorname{HIT}(-\cdot)$. 


\begin{tabular}{|c|c|c|c|c|}
\hline Case & \# elements & $\Delta t[\mu s]$ & \# CPU & hCPU $[\mathrm{kh}]$ \\
\hline DALM & $157 \times 10^{6}$ & 371 & 1120 & 25.4 \\
BF & $284 \times 10^{6}$ & 49 & 1960 & 212.7 \\
HIT, TI $35 \%$ & $139 \times 10^{6}$ & 369 & 1120 & 16.5 \\
\hline
\end{tabular}

Table 4: Computational associated to the different methods of grid turbulence injection.

\subsubsection{Energy spectra}

The mixing and decay regions can be further investigated in terms of transverse, $E_{y y}$ Fig. 15(a), and longitudinal, $E_{x x}$ Fig. 15 (b), frequency 1D power spectra of the velocity fluctuations on the centerline taken at various positions in the flow: $x_{1}, x_{2}, x_{3}, x_{4}$ and $x_{5}$. The temporal signals are gathered during a period of $3 \tau$ with a fixed time-step, $\Delta t l / u_{r e f}=9.4 \times 10^{-7}$.

In the mixing region, at position $x_{1}$, one can observe a frequency peak for both the DALM and the reference case such that $f l / u_{r e f}=0.1-0.2$. This is coherent with the Strouhal number of a single square rod. The spectra obtained with HIT injection have a higher energy level for the large scales since the injected turbulence is already developed with this precursor method.

As for the decay region, for both figures, a downward shift to the large scales, or small frequencies, is observed when advancing in the decay region. The decrease of the area under the spectrum illustrates the decay of the turbulent kinetic energy as expected. For DALM and BF cases, the previously observed eigenfrequency disappear after the destabilization of the grid wake.

For sufficiently large Reynolds numbers the spectra $E_{x x / y y}$ should exhibit a $\sim f^{-5 / 3}$ spectral behavior in the inertial subrange according to Kolmogorov's theory. This trend is observed in the decay region, for $x>x_{3}$, showing sufficiently high Reynolds number and turbulence isotropy, as observed in Fig 13 .

\subsection{Computational cost \& performances}

The computational times needed to reach 15 flow-through times are reported for each case in Table 4. The computational cost of the BF case is almost ten times higher compared to HIT and DALM cases. This is due to the much smaller cells around the discretized grid geometry, requiring a smaller $\Delta t$ to keep a constant CFL number. The cost of DALM and HIT present the same order of magnitude, yet, HIT injection remains at a lower cost. However, the CPU time for HIT doesn't take into account the cost related to the "trial and error" procedure required to find the correct inlet turbulence intensity. This cost can be high depending on the application case. Here, five additional simulations were necessary to correctly tune the turbulence parameters. Moreover, this trial and error procedure is only relevant if the Turbulence Intensity is known accurately: it thus requires either experimental data or empirical correlations. Recall also that the periodic injection of an HIT precursor induces periodicity effects on the flow diagnostics.

\section{Conclusions}

An original method to emulate a turbulence grid in a wind tunnel is proposed. This method is based on the classical Actuator Line Method modified to reproduce realistically bluff body wakes. Fluctuating wakes are obtained using variable aerodynamic coefficients. This method is easy to implement, presents a low computational cost and is independent of the spatial discretization and mesh type.

The method is first applied to the flow over a single square rod. It is compared to the original actuator line method but also with a scale resolving wall-modeled LES which serves as 
a reference case. This comparison shows that the Dynamic Actuator Line Method can generate a realistic wake with vortex shedding downstream the body. The time-averaged and fluctuation velocity profiles are in better agreement with the reference LES compared to those produced by the standard Actuator Line Method.

The method is also assessed by simulating a wind tunnel turbulence grid. To that end, the results of three different strategies are compared to experimental data. Particular attention was paid to the grid wake destabilization process and how the nearly isotropic turbulence state is reached in the wind tunnel. The classical precursor method consisting of injecting homogeneous isotropic turbulence provides correct results, yet it required a time-consuming trial and error search using experimental data to obtain the correct turbulence intensity and its decay along the tunnel. Moreover for specific cases, the anisotropy of the turbulence cannot be emulated by such method. The Body Fitted and the Dynamic Actuator Line Methods can produce the correct turbulence diagnostics at a lower degree of empiricism and present similar destabilization lengths. However, the dynamic actuator line method presents a computational cost that is ten times lower.

However, this method is based on numerous hypothesis, which could be enhanced in future studies. The drag fluctuations are not taken into account since they remain small compared to those of the lift. When more than one object needs to be modeled in the flow, the phase shift has to be taken into account. In the grid study, the phase shift is random. For the grid case, the rod to rod interaction is taken into account only between parallels rods, through aerodynamic coefficients from literature. The "nodes" of the grid mesh are therewith not taken into account in this study.

The method proposed here can be applied to many other problems, especially when the global influence of a body on the flow has to be taken into account and the geometrical details are not important. For instance, the proposed method can be applied to emulate wake passage in turbine cascade [56], wire modeling for boundary layer tripping, active grid turbulence generation, modeling of complex geometrical entities in environmental flows (e.g. pylons, trees, etc.).

\section{Acknowledgments}

This work was granted access to the HPC resources from CRIANN (Centre Régional Informatique et d'Applications Numériques de Normandie) under the allocation 2012006 and from TGCC-CEA under the allocations x20172b6880 made by GENCI (Grand Equipement National de Calcul Intensif). This project was co-financed by the European Union with the European regional development fund (ERDF, HN0005592) and by the Normandy Regional Council via the NEPTUNE project. Part of this work was funded by the Fonds de la Recherche Scientifique de Belgique (F.R.S.-FNRS, Mobility grant for Insa-Rouen).

\section{References}

[1] J. Slotnick, A. Khodadoust, J. Alonso, D. Darmofal, W. Gropp, E. Lurie, and D. Mavriplis, "Cfd vision 2030 study: A path to revolutionary computational aerosciences: Nasa," tech. rep., NASA, 2014.

[2] G. Vita, H. Hemida, T. Andrianne, and C. C. Baniotopoulos, "Generating atmospheric turbulence using passive grids in an expansion test section of a wind tunnel," Journal of Wind Engineering and Industrial Aerodynamics, vol. 178, pp. 91-104, 2018.

[3] J. Hinze, Turbulence, vol. 218. McGraw-Hill, 1975.

[4] G. K. Batchelor, The theory of homogeneous turbulence. Cambridge university press, 1953. 
[5] P. Roach, "The generation of nearly isotropic turbulence by means of grids," International Journal of Heat and Fluid Flow, vol. 8, no. 2, pp. 82-92, 1987.

[6] D. Hurst and J. C. Vassilicos, "Scalings and decay of fractal-generated turbulence," Physics of Fluids, vol. 19, no. 3, pp. 035-103, 2007.

[7] B. Mazzi and J. Vassilicos, "Fractal-generated turbulence," Journal of Fluid Mechanics, vol. 502, pp. 65 87, 032004 .

[8] S. Laizet and J. C. Vassilicos, "Dns of fractal-generated turbulence," Flow, turbulence and combustion, vol. 87, no. 4, pp. 673-705, 2011.

[9] N. S. Dhamankar, G. A. Blaisdell, and A. S. Lyrintzis, "Overview of turbulent inflow boundary conditions for large-eddy simulations," AIAA Journal, vol. 56, no. 4, pp. 1317-1334, 2018.

[10] G. Tabor and M. Baba-Ahmadi, "Inlet conditions for large eddy simulation: A review," Computers \&6 Fluids, vol. 39, no. 4, pp. $553-567,2010$.

[11] A. Keating, U. Piomelli, E. Balaras, and H.-J. Kaltenbach, "A priori and a posteriori tests of inflow conditions for large-eddy simulation," Physics of Fluids, vol. 16, no. 12, pp. 4696-4712, 2004.

[12] L. Davidson, "Hybrid les-rans: Inlet boundary conditions for flows with recirculation," in Advances in Hybrid RANS-LES Modelling (S.-H. Peng and W. Haase, eds.), (Berlin, Heidelberg), pp. 55-66, Springer Berlin Heidelberg, 2008.

[13] M. Klein, A. Sadiki, and J. Janicka, "A digital filter based generation of inflow data for spatially developing direct numerical or large eddy simulations," J. Comput. Phys., vol. 186, pp. 652-665, Apr. 2003.

[14] T. S. Lund, X. Wu, and K. D. Squires, "Generation of turbulent inflow data for spatially-developing boundary layer simulations," Journal of computational physics, vol. 140, no. 2, pp. 233-258, 1998.

[15] J. Mann, "The spatial structure of neutral atmospheric surface-layer turbulence," Journal of Fluid Mechanics, vol. 273, p. 141-168, 1994.

[16] W. Munters, C. Meneveau, and J. Meyers, "Turbulent inflow precursor method with time-varying direction for large-eddy simulations and applications to wind farms," Boundary-layer meteorology, vol. 159, no. 2, pp. 305-328, 2016.

[17] X. Wu, "Inflow Turbulence Generation Methods," Annual Review of Fluid Mechanics, vol. 49, no. 1, pp. 23-49, 2016.

[18] S. K. Arolla, "Inflow turbulence generation for eddy-resolving simulations of turbomachinery flows," Journal of Fluids Engineering, vol. 138, no. 3, p. 031201, 2015.

[19] J. A. Sillero, J. Jiménez, and R. D. Moser, "One-point statistics for turbulent wall-bounded flows at Reynolds numbers up to $\delta+=2000$," Physics of Fluids, vol. 25, no. 10, 2013.

[20] C. S. Peskin, "Flow patterns around heart valves: A numerical method," Journal of Computational Physics, vol. 10, no. 2, pp. 252-271, 1972.

[21] D. Foti, X. Yang, F. Campagnolo, D. Maniaci, and F. Sotiropoulos, "On the use of spires for generating inflow conditions with energetic coherent structures in large eddy simulation," Journal of Turbulence, vol. 18, no. 7, pp. 611-633, 2017.

[22] T. Blackmore, W. M. Batten, and A. S. Bahaj, "Inlet grid-generated turbulence for large-eddy simulations," International Journal of Computational Fluid Dynamics, vol. 27, no. 6-7, pp. 307-315, 2013.

[23] J. Sørensen and W. Shen, "Numerical modeling of wind turbine wakes.," J Fluids Eng, vol. 124, no. 2, pp. 393-399, 2002.

[24] F. Nicoud, H. B. Toda, O. Cabrit, S. Bose, and J. Lee, "Using singular values to build a subgrid-scale model for large eddy simulations," Physics of Fluids, vol. 23, no. 8, pp. 085-106, 2011. 
[25] Y. Peet, P. Fischer, G. Conzelmann, and V. Kotamarthi, "Actuator line aerodynamics model with spectral elements," in 51st AIAA Aerospace Sciences Meeting including the New Horizons Forum and Aerospace Exposition, p. 1210, 2013.

[26] H. Glauert, "Airplane Propellers," Durand, W.F., Ed., Aerodynamic Theory, vol. IV, pp. 169-360, 1935.

[27] P. Benard, A. Viré, V. Moureau, G. Lartigue, L. Beaudet, P. Deglaire, and L. Bricteux, "Large-eddy simulation of wind turbines wakes including geometrical effects," Computers \& Fluids, vol. 173, pp. 133$139,2018$.

[28] N. Troldborg, J. N. Sørensen, and R. Mikkelsen, "Numerical simulations of wake characteristics of a wind turbine in uniform inflow," Wind Energy, vol. 13, p. 86-99, 2010.

[29] L. A. Martínez-Tossas, M. J. Churchfield, and C. Meneveau, "Optimal smoothing length scale for actuator line models of wind turbine blades based on gaussian body force distribution.," Wind Energ., 2017.

[30] V. Moureau, P. Domingo, and L. Vervisch, "Design of a massively parallel cfd code for complex geometries," Comptes Rendus Mécanique, vol. 339, no. 2-3, pp. 141-148, 2011.

[31] M. Kraushaar, Application of the compressible and low-Mach number approaches to Large-Eddy Simulation of turbulent flows in aero-engines. PhD thesis, INPT, 2011.

[32] A. J. Chorin, "Numerical solution of the navier-stokes equations. mathematics of computation," Math Comp, vol. 22, pp. 745-762, 2013.

[33] M. Malandain, N. Maheu, and V. Moureau, "Optimization of the deflated conjugate gradient algorithm for the solving of elliptic equations on massively parallel machines.," J Comput Phys, vol. 238, pp. 32-47, 2013.

[34] V. Moureau, P. Domingo, and L. Vervisch, "From large-eddy simulation to direct numerical simulation of a lean premixed swirl flame: filtered laminar flame-pdf modeling.," Combust Flame, vol. 158, no. 7, pp. 1340-1357, 2011.

[35] F. Lesage and L. Gartshore, "A method of reducing drag and fluctuating side force on bluff bodies.," Journal of Wind Engineering and Industrial Aerodynamics, vol. 25, pp. 229-245, 1987.

[36] C. Knisely, "Strouhal numbers of rectangular cylinders at incidence: a review and new data .," Journal Fluids and Structures, vol. 4, pp. 371-393, 1990.

[37] B. Vickery, "Fluctuating lift and drag on a long cylinder of square cross-section in a smooth and in a turbulent stream.," J. Fluid Mech., vol. 25, no. 3, pp. 481-494, 1966.

[38] H. Fukumoto, H. Aono, M. Tanaka, H. Matsuda, T. Osako, T. Nonomura, A. Oyama, and K. Fujii, "Significance of computational spanwise domain length on LES for the flowfield with large vortex structure," 54th AIAA Aerospace Sciences Meeting, pp. 1-16, 2016.

[39] C. Duprat, G. Balarac, O. Métais, P. M. Congedo, and O. Brugière, "A wall-layer model for large-eddy simulations of turbulent flows with/out pressure gradient," Physics of Fluids, vol. 23, no. 1, 2011.

[40] C. H. Williamson and A. Roshko, "Vortex formation in the wake of an oscillating cylinder," Journal of Fluids and Structures, vol. 2, no. 4, pp. 355-381, 1988.

[41] L. Guedot, G. Lartigue, and V. Moureau, "Design of high-order implicit filters on unstructured grids for the identification of large-scale features in large-eddy simulations," Direct and Large-Eddy Simulation IX, vol. 20, pp. 81-87, 2015.

[42] N. Legrand, G. Lartigue, and V. Moureau, "A multi-grid framework for the extraction of large-scale vortices in Large-Eddy Simulation," Journal of Computational Physics, vol. 349, pp. 528-560, 2017.

[43] G. W. Jones, J. J. Cincotta, and W. R. W., "Aerodynamic forces on a stationary and oscillating circular cylinder at high Reynolds numbers," NASA technichal report, 1969.

[44] L. Fiabane, M. Gohlke, and O. Cadot, "Characterization of flow contributions to drag and lift of a circular cylinder using a volume expression of the fluid force," European Journal of Mechanics - B/Fluids, vol. 30, no. 3, pp. 311-315, 2011. 
[45] J. Zhao, K. Hourigan, and M. C. Thompson, "Flow-induced vibration of D-section cylinders: An afterbody is not essential for vortex-induced vibration," Journal of Fluid Mechanics, vol. 851, pp. 317-343, 2018.

[46] F. Mühle, J. Schottler, J. Bartl, R. Futrzynski, S. Evans, L. Bernini, P. Schito, M. Draper, A. Guggeri, E. Kleusberg, et al., "Blind test comparison on the wake behind a yawed wind turbine," Wind Energy Science, vol. 3, no. 2, pp. 883-903, 2018.

[47] L. Sætran, F. Mühle, J. Bartl, J. Schottler, M. Hölling, and M. S. Adaramola, "Invitation to the 2017 "blind test 5" workshop - the wake behind a yawed wind turbine.," http://doi.org/10.5281/zenodo.1218555, 2018.

[48] M. M. Alam, Y. Zhou, and X. Wang, "The wake of two side-by-side square cylinders," Journal of Fluid Mechanics, vol. 669, pp. 432-471, 2011.

[49] R. Martinuzzi, S. Bailey, and G. Kopp, "Influence of wall proximity on vortex shedding from a square cylinder," Experiments in fluids, vol. 34, no. 5, pp. 585-596, 2003.

[50] D. Chatterjee and G. Biswas, "Dynamic behavior of flow around rows of square cylinders kept in staggered arrangement," Journal of Wind Engineering and Industrial Aerodynamics, vol. 136, pp. 1-11, 2015.

[51] T. Passot and A. Pouquet, "Numerical simulation of compressible homogeneous flows in the turbulent regime," Journal of Fluid Mechanics, vol. 181, p. 441-466, 1987.

[52] U. Ciri, G. Petrolo, M. V. Salvetti, and S. Leonardi, "Large-eddy simulations of two in-line turbines in a wind tunnel with different inflow conditions," Energies, vol. 10, pp. 1-23, 2017.

[53] S. Laizet and J. C. Vassilicos, "DNS of fractal-generated turbulence," Flow, Turbulence and Combustion, vol. 87, no. 4, pp. 673-705, 2011.

[54] J. L. Lumley and G. R. Newman, "The return to isotropy of homogeneous turbulence," Journal of Fluid Mechanics, vol. 82, no. 1, p. 161-178, 1977.

[55] S. Banerjee, R. Krahl, F. Durst, and C. Zenger, "Presentation of anisotropy properties of turbulence, invariants versus eigenvalue approaches," Journal of Turbulence, vol. 8, no. December 2014, pp. 1-27, 2007.

[56] X. Qu, Y. Zhang, X. Lu, and J. Zhu, "Unsteady effects of periodic wake passing frequency on aerodynamic performance of ultra-high-lift low pressure turbine cascades," Physics of Fluids, vol. 31, no. 9, pp. 094-102, 2019. 